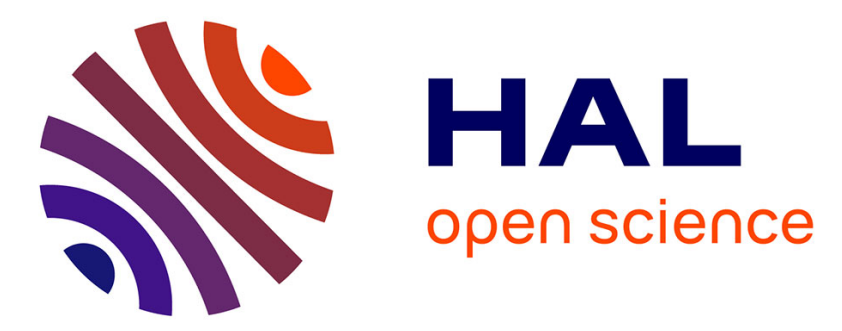

\title{
Prediction of Separation-induced Transition on the SD7003 Airfoil using Algebraic Transition Triggering
}

Luis Bernardos, François Richez, Vincent Gleize, Georges Gerolymos

\section{To cite this version:}

Luis Bernardos, François Richez, Vincent Gleize, Georges Gerolymos. Prediction of Separationinduced Transition on the SD7003 Airfoil using Algebraic Transition Triggering. AIAA Journal, 2019, 57 (9), pp.3812-3824. 10.2514/1.J058288 . hal-02294755

\section{HAL Id: hal-02294755 \\ https://hal.science/hal-02294755}

Submitted on 23 Sep 2019

HAL is a multi-disciplinary open access archive for the deposit and dissemination of scientific research documents, whether they are published or not. The documents may come from teaching and research institutions in France or abroad, or from public or private research centers.
L'archive ouverte pluridisciplinaire HAL, est destinée au dépôt et à la diffusion de documents scientifiques de niveau recherche, publiés ou non, émanant des établissements d'enseignement et de recherche français ou étrangers, des laboratoires publics ou privés. 


\title{
Prediction of Separation-induced Transition on the
}

\section{SD7003 Airfoil using Algebraic Transition Triggering}

\author{
Luis Bernardos ${ }^{1}$, F. Richez ${ }^{2}$ and V. Gleize ${ }^{3}$ \\ ONERA, Meudon, 92190, France \\ G.A. Gerolymos ${ }^{4}$ \\ Sorbonne Université, 75005 Paris, France
}

The paper investigates the performance of a recently developed laminar separation transition triggering (LSTT) approach (criteria-based) which controls the transitional turbulence source terms. Computational results are compared with available experimental and LES data for low-Reynolds number $\left(\mathbf{1 0 , 0 0 0}<R e_{c}<\mathbf{9 0 , 0 0 0 )}\right.$ quasiincompressible flow over the SD7003 airfoil. The influence of angle of attack, chordbased Reynolds number, and turbulence intensity at transition onset is satisfactory provided that the correct transition location is returned by the correlations. The results highlight the importance of correctly modeling the rapid growth of turbulence kinetic energy immediately downstream of transition onset.

Nomenclature

$\begin{array}{ll}c & =\text { airfoil chord length }(\mathrm{m}) \\ C_{\lim } & =\text { shear-stress limiter delay function } \\ C_{p} & =\text { pressure coefficient } \\ c_{f_{\infty}} & =\text { skin-friction coefficient }\left(\tau_{w} / \frac{1}{2} \rho_{\infty} U_{\infty}^{2}\right) \\ F_{2} & =\text { Menter's second blending function }\end{array}$

1 PhD Student, Aerodynamics, Aeroelasticity and Acoustics Department. luis.bernardos_barreda@onera.fr

2 Research Scientist, Aerodynamics, Aeroelasticity and Acoustics Department. francois.richez@onera.fr

3 Research Scientist, Aerodynamics, Aeroelasticity and Acoustics Department. vincent.gleize@onera.fr

4 Professor, Faculty of Science and Engineering, 4 place Jussieu, Senior Member AIAA. georges.gerolymos@sorbonneuniversite.fr 


$$
\begin{aligned}
& f^{\mathrm{tr}} \quad=\text { Transition function } \\
& H_{k} \quad=\text { boundary-layer kinematic shape factor }\left(=\delta_{k}^{*} / \theta_{k}\right) \\
& k \quad=\text { turbulence kinetic energy }\left(\mathrm{m}^{2} \mathrm{~s}^{-2}\right) \\
& k_{\mathrm{L}} \quad=\text { laminar kinetic energy }\left(\mathrm{m}^{2} \mathrm{~s}^{-2}\right) \\
& M \quad=\text { Mach number } \\
& N \quad=\text { total amplification factor } \\
& N_{c r} \quad=\text { critical total amplification factor } \\
& n \quad=\text { airfoil wall-normal coordinate }(\mathrm{m}) \\
& \mathcal{P}_{k} \quad=\text { production of turbulence kinetic energy }\left(\mathrm{kg} \mathrm{m}^{-1} \mathrm{~s}^{-3}\right) \\
& p \quad=\text { static pressure }(\mathrm{Pa}) \\
& R e_{c} \quad=\text { chord-based Reynolds number, } \rho_{\infty} c U_{\infty} / \mu_{\infty} \\
& \operatorname{Re}_{\theta} \quad=\text { momentum-thickness Reynolds number, } \rho_{e} \theta U_{e} / \mu_{e} \\
& \bar{S}_{i j} \quad=\text { mean strain rate tensor }\left(\mathrm{s}^{-1}\right) \\
& s \quad=\text { wall-tangent curvilinear abscissa }(\mathrm{m}) \\
& s_{\mathrm{sep}} \quad=\text { laminar separation point }(\mathrm{m}) \\
& s_{\mathrm{tr}} \quad=\text { transition onset }(\mathrm{m}) \\
& \hat{s}=\text { Dimensionless streamwise abcissa, }\left(s-s_{\mathrm{tr}}\right) /\left(s_{\mathrm{tr}}-s_{\mathrm{sep}}\right) \\
& \hat{s}_{a}, \hat{s}_{b}, \hat{s}_{c}, a=\text { Transitional model closure coefficients } \\
& T_{u} \quad=\text { turbulence intensity, }(2 k / 3)^{1 / 2} / U_{e} \\
& \bar{u}_{i} \quad=\text { local Reynolds-averaged velocity } i \text {-component }\left(\mathrm{m} \mathrm{s}^{-1}\right) \\
& u_{s} \quad=\text { velocity component projected onto } s \text { direction }\left(\mathrm{m} \mathrm{s}^{-1}\right) \\
& U_{e} \quad=\text { boundary-layer edge velocity }\left(\mathrm{m} \mathrm{s}^{-1}\right) \\
& U_{\infty} \quad=\text { inflow freestream velocity }\left(\mathrm{m} \mathrm{s}^{-1}\right) \\
& \alpha \quad=\text { airfoil's angle of attack (deg) } \\
& \alpha_{\omega} \quad=\text { turbulence model's production of } \omega \text { closure coefficient } \\
& \beta_{k}, \beta_{\omega} \quad=\text { turbulence model's dissipation of } k \text { and } \omega \text { closure coefficients } \\
& \gamma \quad=\text { Intermittency factor } \\
& \delta \quad=\text { boundary-layer thickness }(\mathrm{m}) \\
& \delta^{*}=\text { boundary-layer displacement thickness }(\mathrm{m}) \\
& \varepsilon \quad=\text { dissipation of turbulence kinetic energy }\left(\mathrm{m}^{2} \mathrm{~s}^{-3}\right) \\
& \theta \quad=\text { boundary-layer momentum thickness }(\mathrm{m}) \\
& \mu \quad=\text { molecular dynamic viscosity }\left(\mathrm{kg} \mathrm{m}^{-1} \mathrm{~s}^{-1}\right)
\end{aligned}
$$




$$
\begin{array}{ll}
\mu_{t} & =\text { dynamic eddy viscosity }\left(\mathrm{kg} \mathrm{m}^{-1} \mathrm{~s}^{-1}\right) \\
\nu & =\text { molecular kinematic viscosity }\left(\mathrm{m}^{2} \mathrm{~s}^{-1}\right) \\
\nu_{t} & =\text { kinematic eddy viscosity }\left(\mathrm{m}^{2} \mathrm{~s}^{-1}\right) \\
\rho & =\text { density }\left(\mathrm{kg} \mathrm{m}{ }^{-3}\right) \\
\sigma_{k}, \sigma_{\omega}, \sigma_{d} & =\text { turbulence model's Prandtl diffusion coefficients for } k, \omega, \text { and the cross-diffusion. } \\
\tau_{w} & =\text { wall shear stress }\left(\mathrm{kg} \mathrm{m}^{-1} \mathrm{~s}^{-2}\right) \\
\omega & =\text { Turbulence specific dissipation rate }\left(\mathrm{s}^{-1}\right)
\end{array}
$$

\section{Introduction}

ANALYSIS and design of low Reynolds number airfoils [1] is essential in numerous applications, including unmanned aerial vehicles (UAVs) or radio-controlled (RC) model aircraft [2], micro-air vehicles (MAVs) [3] and small horizontal-axis wind turbines (HAWTs) [4].

Advances in low-Reynolds airfoil design were made mainly by recognizing the major influence of the upper-surface laminar separation bubble (LSB) in the boundary-layer development and consequently on overall airfoil performance. Selig [1] and coworkers [5], extensively applied inverse design prescribing the boundary-layer behavior to produce several very successful and widely used low-Reynolds airfoils. An important element in this design philosophy is based on the observation [6] that low-Reynolds airfoil drag essentially depends on the location of transition (optimally inside a short LSB) as a function of angle of attack (or lift-coefficient $c_{L}$ ), which is prescribed as target in the inverse method. Therefore, tools that accurately predict transition inside LSBs are necessary.

Traditional viscous/inviscid methods [7] based on integral boundary-layer methods [8] and transition correlations [9] are quite successful in predicting 2D airfoil flows. On the other hand, the development of more general, potentially extensible to 3D, analysis and design tools requires the inclusion of transition models in transport-equation RANS (Reynolds-averaged Navier-Stokes) models which are used in CFD [10-14].

Although low-Reynolds multiequation turbulence models were originally introduced to predict transition [10], it is now established that they are not sufficiently precise [13-16]. Therefore various specific transition models have been introduced for use in conjunction with RANS models [17]. These 
models include abrupt activation of production terms in the turbulence transport equations at the correlation-determined transition location $[11,18]$ and specific transport equations for intermittency [19-21] or the laminar kinetic energy [22].

The abrupt activation of turbulence production at the point of predicted transition onset has been justified [11] on the basis that the breakdown to turbulence occurs quite fast. The growth rate of turbulence from the transition onset is of little importance for high Reynolds number flows, as it occurs typically within a small fraction of the airfoil chord. On the contrary, this may have a large impact on low-Reynolds number flows LSBs, as the distance from transition onset up to fully developed turbulence is much longer. As a consequence, the convection effects in the boundarylayer due to the pre-turbulent region are quite significant for low-Reynolds number flows. Lian and Shyy [23] have introduced, in conjunction with the low-Reynolds $k-\omega[10]$ a streamwisedependent intermittency function $\gamma(s)$ to simulate the last stage of the transition process. The lengthscale used in the definition of $\gamma(s)$ was the distance between the transition onset $\left(s_{\text {tr }}\right)$ and separation $\left(s_{\mathrm{sep}}\right)$. Detailed LSB measurements [24] substantiate the idea of using the characteristic lengthscale $s_{\mathrm{tr}}-s_{\mathrm{sep}}$. Bounding $\gamma(s) \leq 1$ retards somehow the streamwise increase of turbulence kinetic energy. Arnal [25, pp. 8-32] suggests the use of a pseudo-intermittency empirical coefficient which can locally overshoot above unity to obtain a rapid growth-rate of turbulence between the transition onset and the complete breakdown to fully turbulent flow. Recently, the authors [26] have developed a laminar separation transition triggering (LSTT) model, based on the lengthscale $s_{\mathrm{tr}}-s_{\mathrm{sep}}$ with a pseudo-intermittency which is locally greater than unity. This approach was coupled with transition criteria $[27,28]$ and different $k-\omega$ variants [29-32]. LSTT model coefficients were calibrated against DNS data [33], to obtain the correct reattachment location. It was found that correctly boosting turbulence production in the transitional region is the key to the successful prediction of the boundary-layer development. In the present work we systematically assess the LSTT model's [26] performance.

The SD7003 airfoil [34] is characterized by a very thin LSB with little influence on airfoil drag, which was attributed [1] to the specific evolution of the upper surface transition location. At low $R e_{c} \in[200 \mathrm{k}, 500 \mathrm{k}]$ laminar separation occurs on the upper surface at a location varying from 
$x_{\mathrm{sep}} / c \in[0.5,0.7]$ for $\alpha=0^{\circ}$ to very near the leading-edge at $\alpha=8^{\circ}$. The transition location $x_{\operatorname{tr}} / c$ varies almost linearly from near the trailing-edge at $\alpha=0^{\circ}$ to $\sim 5 \%$ at $\alpha=8^{\circ}$. Selig [1] attributed the enhanced performance of the SD7003 airfoil with precisely this quasilinear (shallow) evolution of $x_{\mathrm{tr}}$ with $\alpha$ and hence $c_{L}$. It is noteworthy that for $R e_{c} \gtrsim 200 \mathrm{k}$ the SD7003 airfoil does not present any substantial increase of $c_{D}$ at low $c_{L}$ contrary to most other airfoils, this effect appearing at lower $R e_{c} \lesssim 100 \mathrm{k}[34$, Fig. 12.138, p. 332$]$. The LSB on the SD7003 airfoil has been studied extensively for both experimentally [35-41] and computationally using LES [42-48].

Detailed velocimetry [35-37, 39] and skin friction $[49,50]$ measurements, experimental scatter [40], accuracy [37] and interpretation difficulties [1] notwithstanding, provide data on the locations of separation, transition and reattachment (or open bubble) with varying $\left(R e_{c}, \alpha\right)$. The LES computations of Catalano and Tognaccini [43] and Galbraith and Visbal [44] are quite consistent one with another, the observed scatter between computations exemplifying the high sensitivity of the transitional flow which was also observed in the experimental data $[1,37,41,50]$. Several experimental studies $[35,38,50]$ investigate the influence of external flow turbulence intensity of the transition onset station $T_{u_{\mathrm{tr}}}$ on the LSB. Recently Schmidt and Breuer [46] used a specific turbulence generation methodology [47] in the context of LES computations to analyze the effect of $T_{u_{\mathrm{tr}}}$. The available experimental and LES data have been used by numerous authors $[11,12,18,23,51,52]$ to assess the performance of RANS and transitional RANS models.

The purpose of the paper is to further explore the idea of progressive (streamwise) control of the transition process by combining the LSTT model [26] applied to the Wilcox 2006 [53] $k-\omega$ model through systematic validation with available data for quasi-incompressible flow around the SD7003 airfoil. In $\S I I$ we present the physical models and numerical methods employed in this study. In $\S$ III we present the validation results of the LSTT model. A preliminary analysis of the impact of the critical total amplification factor $N_{c r}$ on the transition onset location is performed. Then, the LSTT model is evaluated for varying angles of attack, chord-based Reynolds number and external turbulence level. 


\section{Methodology}

The LSTT approach [26] triggers turbulence production (§II C) in the $k-\omega$ model (§II A) using information on the transition onset location from nonlocal transition criteria (§II B).

\section{A. Turbulence model}

The underlying RANS model which is active in the fully turbulent regions is Wilcox's $2006 k-\omega$ model [32, 53] (Tab. 2)

$$
\begin{aligned}
\frac{\partial \rho k}{\partial t}+\frac{\partial \rho k \bar{u}_{j}}{\partial x_{j}} & =f^{\operatorname{tr}}\left(-\rho \overline{u_{i}^{\prime} u_{j}^{\prime}}\right) \frac{\partial \bar{u}_{i}}{\partial x_{j}}-\beta_{k} \rho \omega k+\frac{\partial}{\partial x_{j}}\left[\left(\mu+\sigma_{k} \mu_{t}\right) \frac{\partial k}{\partial x_{j}}\right] \\
\frac{\partial \rho \omega}{\partial t}+\frac{\partial \rho \omega \bar{u}_{j}}{\partial x_{j}} & =\min \left(f^{\operatorname{tr}}, 1\right) \alpha_{\omega} \frac{\omega}{k}\left(-\rho \overline{u_{i}^{\prime} u_{j}^{\prime}}\right) \frac{\partial \bar{u}_{i}}{\partial x_{j}}-\beta_{\omega} \rho \omega^{2}+\frac{\partial}{\partial x_{j}}\left[\left(\mu+\sigma_{\omega} \mu_{t}\right) \frac{\partial \omega}{\partial x_{j}}\right] \\
& +\sigma_{d} \frac{\rho}{\omega} \max \left(\frac{\partial k}{\partial x_{j}} \frac{\partial \omega}{\partial x_{j}}, 0\right) \\
\left(-\rho u_{i}^{\prime} u_{j}^{\prime}\right) & \approx 2 \mu_{t} \bar{S}_{i j}^{d}-\frac{2}{3} \rho k \delta_{i j} \\
\mu_{t} & =\frac{\rho k}{\max \left\{\omega ; C_{\lim } \sqrt{\frac{2 \bar{S}_{i j}^{d} \bar{S}_{i j}^{d}}{\beta_{k}}}\right\}}
\end{aligned}
$$

where $\bar{S}_{i j}^{d}$ is the deviatoric part of the strain-rate tensor. The LSTT formulation [26] accounts for the separation-induced transition from laminar to turbulent regime by streamwise-variable transition triggering functions (dimensionless function $f^{\text {tr }}$; Fig. 1) multiplying the production terms of the $k$ and $\omega$ equations $[26,54]$, and a delay off-on mechanism in the activation of the shear limiter $C_{\text {lim }}$ (Fig. 1) in the expression of the eddy viscosity (Eq. 1d). Some formulation of shear-stress limiter in the turbulent boundary layer is used in most $k-\omega$ models $[30,32,55,56]$, but this delays transition by reducing the shear-stress and hence the production of $k$ in the transitional region [54]. Turbulence triggering is controlled through both $f^{\text {tr }}$ and a delayed activation of $C_{\lim }(\S \mathrm{II} \mathrm{C})$. The transition triggering functions depend on 2 inputs, the streamwise location of laminar separation $s_{\text {sep }}$ (which is directly obtained from the solution of the Navier-Stokes equations) and the point of transition onset $s_{\text {tr }}$ which is obtained from streamwise-nonlocal criteria based on integral boundary-layer parameters $(\S I I B)$. 


\section{B. Transition onset prediction}

Transition onset is determined by applying the combined [9, 57] AHD [27] and Gleyzes [28] criteria determining the transition-onset location at each iteration of the RANS solver. For the separation-induced transition calculations presented in this paper transition onset is actually determined by the Gleyzes criterion [28]. The AHD criterion is used from the laminar critical point up to the streamwise location where the boundary-layer shape factor $H=2.8$, where it switches to the the Gleyzes criterion. The Gleyzes criterion calculates a total amplification factor $N(s)$ by streamwise integration of correlations based on $H$ and $R e_{\theta}$, which were obtained by fitting linear stability analysis calculations [28]. Transition onset is the location where $N$ reaches a critical value $N_{c r}$. In principle, this critical total amplification factor $N_{c r}$ can be related via Mack's [58] empirical correlation

$$
N_{c r}=-2.4 \ln \left(T_{u_{\mathrm{tr}}}\right)-8.43
$$

to the turbulence intensity, $T_{u_{\mathrm{tr}}}$, at the boundary-layer edge, at the transition onset location. Further details on the implementation of the criteria-based approach $[9,57]$ were presented in the appendix of $[26]$.

This is a quite standard and widely tested criteria-based approach $[9,57]$ numerically similar to the correlations used in XFoil $[8,59]$. Notice that Mack's correlation was developed for low$T_{u}$ studies, and returns $N_{c r}\left(T_{u_{t r}} \gtrsim 2.98 \%\right) \leq 0$. Therefore Drela slightly modifies Eq. (2) at high $T_{u}[60]$. This modification notwithstanding, it should be stated from the outset that a major drawback of Mack's correlation is that it does not include the external turbulence lengthscale, which determines the effectiveness of the external flow perturbations in triggering bypass transition in the boundary-layer $[61,62]$. The sensitivity of results on $N_{c r}$ is further discussed in $\S I I I D$.

\section{Turbulence triggering model}

\section{Off-on triggering}

For comparison purposes we also present results of a simple off-on triggering of the turbulence model. This widely used approach [11], hereafter denoted step, consists in disabling the source terms of the turbulence model upstream of the transition onset location where they are instantly 
activated. The step approach was evaluated both with the shear stress limiter enabled $\left(C_{\lim }=7 / 8\right)$ and disabled $\left(C_{\lim }=0\right)$.

\section{2. $L S T T$}

The current work assesses by systematic comparison with measurements the non-local algebraic laminar separation transition triggering (LSTT) model recently developed by the authors [26]. Offon abrupt triggering of the RANS model at transition onset underestimates the rapid increase of skin-friction downstream of the bubble [54], compared to DNS data [33] where $c_{f}$ increases rapidly and presents an overshoot just downstream of reattachment. To properly handle this behaviour, the triggering model must account for the smooth but swiftly increasing streamwise production of the Reynolds shear stress, $-\overline{u^{\prime} v^{\prime}}$, that occurs in the separated shear layer, downstream of transition onset, as observed both in experiments [39] and DNS [33]. The LSTT model [26] was specifically designed to control the rate at which turbulence is produced downstream of the transition onset. Proper growth of the Reynolds shear stress is accomplished by two mechanisms: (a) favoring a strong imbalance of production-to-dissipation ratio of turbulence kinetic energy in the transitional region by boosting the production of $k$ with a streamwise-variable multiplicative coefficient $f^{\operatorname{tr}}(s)$ (Eq. 1a) which is locally $>1$, whereas the production of $\omega$ grows without overshoot (Eq. 1b) by limiting the multiplicative coefficient to $\min \left(f^{\text {tr }}, 1\right)$, and (b) delaying the activation of the shear limiter $C_{\lim }$ until a fully turbulent boundary-layer is established.

In constructing the model it was assumed (Fig. 1) that the transitional region streamwise extent scales with the bubble's laminar length, $s_{\mathrm{tr}}-s_{\mathrm{sep}}$, and, furthermore, that the triggering functions, $f^{\operatorname{tr}}(s)$ and $C_{\lim }(s)$, depend only on the nondimensional streamwise coordinate

$$
\hat{s}:=\frac{s-s_{\mathrm{tr}}}{s_{\mathrm{tr}}-s_{\mathrm{sep}}}
$$

In this way turbulence triggering automatically adapts to different bubble sizes. For the airfoil case, $s$ is the curvilinear coordinate along the airfoil surface.

In the LSTT model [26] $f^{\operatorname{tr}}(\hat{s})$ is a piecewise continuous function (Fig. 1) that depends on 3 parameters, the overshot value $a:=\max _{s}\left(f^{\text {tr }}\right)$, the nondimensional location $\hat{s}_{a}$ of this maximum, and the downstream nondimensional station $\hat{s}_{b}$ where $f^{\operatorname{tr}}\left(\hat{s}_{b}\right)=1$. The model is completed by the 


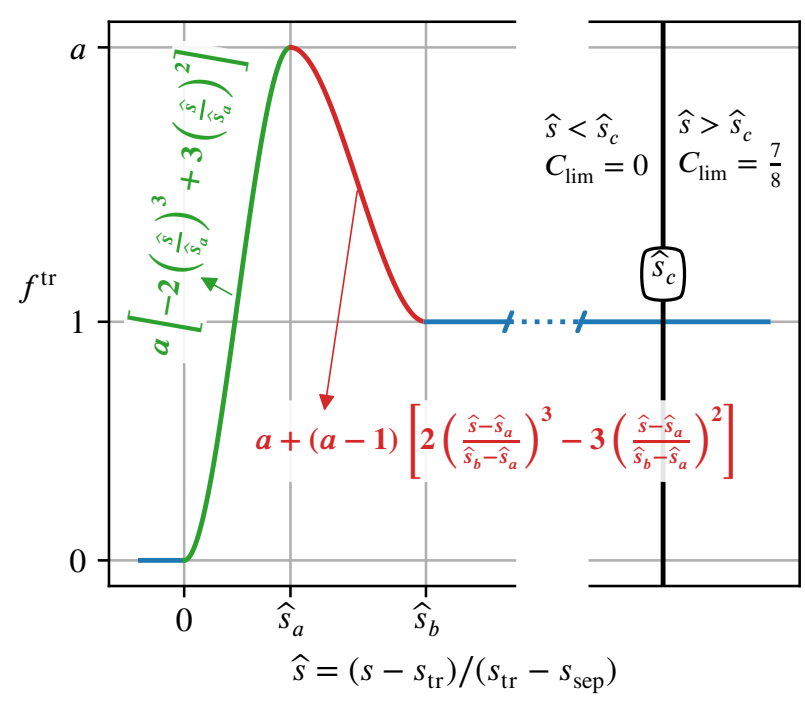

Fig. 1 Transition-triggering function $f^{\text {tr }}$ and shear stress limiter $C_{\text {lim }}$ activation used in the LSTT [26] approach for the Wilcox $2006 k-\omega$ model (Tab. 2).

nondimensional station where the shear limiter $C_{\lim }$ is activated further downstream, $\hat{s}_{c}$, typically several bubble-lengths downstream of the bubble. These 4 model coefficients $\left(a, \hat{s}_{a}, \hat{s}_{b}, \hat{s}_{c}\right)$ were calibrated [26] for different $k-\omega$ models to reproduce the DNS-calculated [33] $c_{f}$ distribution of the LSB on a flat-plate. The specific values for the Wilcox $2006 k-\omega$ model which were used in the present computatons are summarized in Tab. 2. The required inputs of the LSTT model are the laminar separation point $s_{\text {sep }}$, obtained by the solution of the Navier-Stokes equations at the current iteration, and the transition onset location $s_{t r}$ provided by the transition criteria (§II B).

Table 2 Wilcox (2006) $k-\omega$ [53] turbulence model closure coefficients (notice that the 3-D term $f_{\beta_{\omega}}=1$ for 2-D flows) and corresponding LSTT [26] model coefficients (Fig. 1).

\begin{tabular}{ccccccccccc}
\hline \hline$\beta_{k}$ & $\sigma_{k}$ & $\alpha_{\omega}$ & $\beta_{\omega}$ & $\sigma_{\omega}$ & $\sigma_{d}$ & $C_{\lim }$ & $\hat{s}_{a}$ & $\hat{s}_{b}$ & $\hat{s}_{c}$ & $a$ \\
\hline 0.09 & 0.6 & 0.52 & $0.0708 f_{\beta_{\omega}}$ & 0.5 & 0.125 & $\frac{7}{8} ; s \geq s_{c}$ & 0.182 & 0.414 & 5.83 & 2.15 \\
0 & $0 ; s<s_{c}$ \\
\hline
\end{tabular}

3. 2-D extrusion

The LSTT model acts through the algebraic functions $f^{\text {tr }}$ and $C_{\lim }$ in the $k-\omega$ Eqs. 1 . Therefore, these functions which are determined at the wall are required everywhere in the flowfiled. 
Inside the boundary-layer ( $n \leq \delta(s)$, where $n$ is the distance to the wall) they are determined by wallnormal extrusion. Outside of the boundary-layer $(n>\delta(s))$, a simple off-on switch of $f^{\text {tr }} \in\{0,1\}$ and $C_{\text {lim }} \in\left\{0, \frac{7}{8}\right\}$ is made in the laminar boundary-layer edge region $\left(s \leq s_{t r}\right)$.

\section{Flow solver}

Computations were run on structured multiblock grids using the compressible solver elsA [63] developed at ONERA. The freestream Mach number, $M_{\infty}=0.16$, corresponds to essentially incompressible flow. The turbulence kinetic energy production was limited to $\max \left(\mathcal{P}_{k}, 100 \varepsilon\right)$. This limiter is active in the transitional region where extreme non-equilibrium may be returned by the model, as shown in the Appendix.

Details on the numerical method and boundary-conditions are given in [26]. Following standard computational practice [64] the freestream values $\mu_{t_{\infty}}=0.1 \mu$ and $T_{u_{\infty}}=0.01 \%$ were applied. The corresponding lengthscale is $\ell_{t_{\infty}}:=\sqrt{k_{\infty}} / \beta_{k} / \omega_{\infty} \approx 9000 c / R e_{c}$. In the region $s(x, y)<s_{t r}$, where $f^{\text {tr }}=0$, there is no production in the $k-\omega$ Eqs. $(1 \mathrm{a}, 1 \mathrm{~b})$, and the model behaves as decaying turbulence [32,65]. Since $\nu_{t_{\infty}} \ll \nu_{\infty}$ the code returns essentially laminar results up to $s_{\mathrm{tr}}$.

To thoroughly investigate the influence of $T_{u_{\mathrm{tr}}}$ and the applicability of Mack's [58] correlation (Eq. 2), $N_{c r}$ is prescribed as an independent input, in lieu of using the computed value of $T_{u_{\delta}}\left(s_{\mathrm{tr}}\right)$, thus circumventing well known issues of the farfield boundary-condition of the $k-\omega$ model [64].

\section{E. Grid assessment}

The C-topology grids were generated by 2-D hyperbolic extrusion using Cassiopee [66]. The results (§III) were obtained on the $867 \times 176$ points grids (Tab. 3) designed to have similar resolution as the grids used by Catalano and Tognaccini [18], with the farfield boundary located at 30 chords from the airfoil, and the wall-adjacent cell height $\Delta n_{w}^{+}<1$ in the turbulent boundary-layer. In the streamwise direction, the $867 \times 176$ points grids have 96 points in the wake, 179 points on the lower side of the airfoil, and 500 points on the upper side. Depending of the computed $\left(\alpha, R e_{c}\right)$-case, the number of streamwise points on the upper side of the airfoil upstream of separation $N_{s<s_{\text {sep }}}$, $N_{\mathrm{LSB}}$ within the LSB, and $N_{s>s_{\text {reat }}}$ from reattachment to the trailing edge varied. Invariably the LSB was finely relsolved $\left(N_{\mathrm{LSB}} \in[177,320]\right)$ with $N_{\delta}\left(s_{\mathrm{sep}}\right) \in[61,91]$ wall-normal points across the 
Table 3 Computational grids and LSB resolution parameters (based on the $k-\omega+$ LSTT [26] results).

\begin{tabular}{|c|c|c|c|c|c|c|}
\hline$\alpha\left(^{\circ}\right)$ & $R e_{c}$ & $N_{i} \times N_{j}$ & $\begin{array}{c}N_{s<s_{\text {sep }}} \\
0<x<x_{\text {sep }}\end{array}$ & $\begin{array}{c}N_{\mathrm{LSB}} \\
x_{\text {sep }}<x<x_{\text {reat }}\end{array}$ & $\begin{array}{l}N_{s>s_{\text {reat }}} \\
x_{\text {reat }}<x\end{array}$ & $N_{\delta}\left(s_{\mathrm{sep}}\right)$ \\
\hline 2 & $60 \times 10^{3}$ & $867 \times 176$ & 153 & 318 & 22 & 78 \\
\hline 4 & $10 \times 10^{3}$ & & 173 & 320 & 0 & 91 \\
\hline 4 & $40 \times 10^{3}$ & & 105 & 301 & 87 & 79 \\
\hline 4 & $60 \times 10^{3}$ & & 115 & 268 & 110 & 76 \\
\hline 6 & $60 \times 10^{3}$ & & 51 & 188 & 254 & 73 \\
\hline 8 & $30 \times 10^{3}$ & & 50 & 203 & 240 & 74 \\
\hline 8 & $60 \times 10^{3}$ & & 39 & 177 & 277 & 68 \\
\hline 8 & $90 \times 10^{3}$ & & 43 & 200 & 250 & 65 \\
\hline 11 & $60 \times 10^{3}$ & & 43 & 196 & 254 & 61 \\
\hline \multirow[t]{5}{*}{4} & $60 \times 10^{3}$ & $434 \times 89$ & 79 & 118 & 50 & 40 \\
\hline & & $434 \times 176$ & 57 & 136 & 54 & 76 \\
\hline & & $867 \times 351$ & 108 & 291 & 94 & 151 \\
\hline & & $1734 \times 176$ & 231 & 541 & 217 & 76 \\
\hline & & $1734 \times 351$ & 217 & 582 & 190 & 151 \\
\hline
\end{tabular}

boundary-layer at separation (Tab. 3).

A grid convergence study is shown in Fig. 2, where the skin-friction coefficient, $c_{f_{\infty}}=$ $\tau_{w} / \frac{1}{2} \rho_{\infty} U_{\infty}^{2}$, and pressure coefficient, $C_{p}=\left(p-p_{\infty}\right) / \frac{1}{2} \rho_{\infty} U_{\infty}^{2}$, are presented for the baseline SD7003 case $\left(\alpha=4^{\circ}, R e_{c}=60,000\right)$. This study highlights the importance of wall-normal resolution, as the coarsest $434 \times 89$ point grid produces inaccurate results, contrary to the $434 \times 176$ points grid. The finest wall-normalwise grids, $867 \times 351$ and $1734 \times 351$, yield almost identical results (Fig. 2), which can be considered grid-converged. The $867 \times 176$ grid is in quite good agreement with the grid-converged results, and was retained for the systematic comparison of the LSTT model [26] with $\operatorname{data}(\S \mathrm{III})$. 

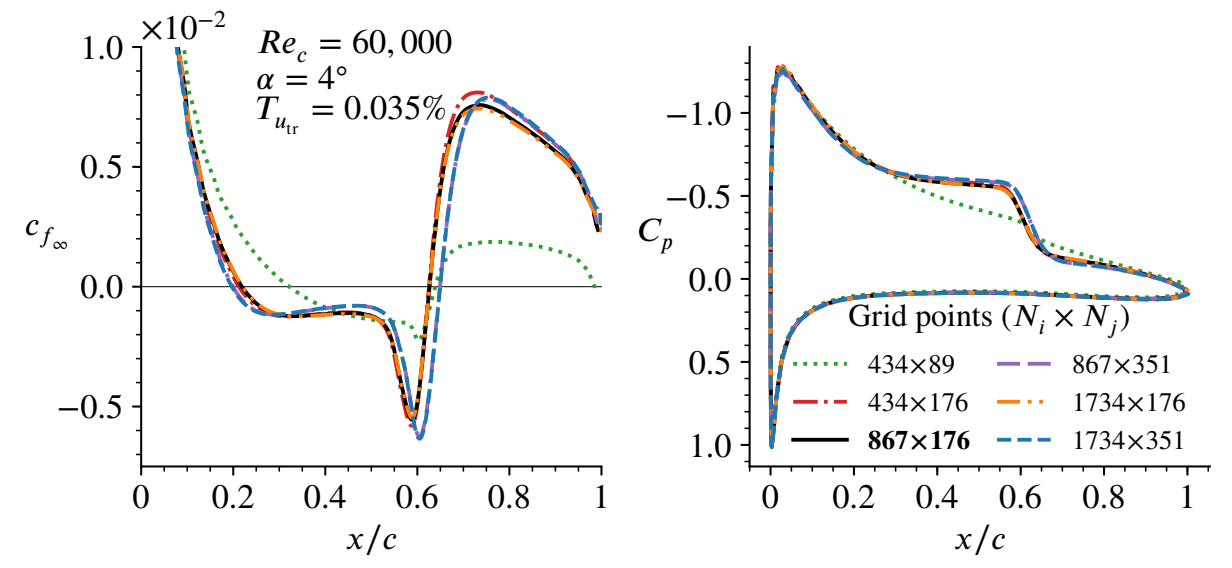

Fig. 2 Distributions of $c_{f_{\infty}}$ (on the suction side) and $C_{p}$ using $k-\omega$ [53] + LSTT [26] of the flow around a SD7003 airfoil on progressively refined grids (Tab. 3).

\section{Results and discussion}

After a detailed study of the model for the baseline $\left(\alpha=4^{\circ}, R e_{c}=60,000\right)$ configuration of the SD7003 airfoil (§III A), we assess the method's performance in predicting the effects of angle of attack (§IIIB), Reynolds number (§III C) and ambient turbulence (§IIID).

A. SD7003: $\alpha=4^{\circ}$ at $R e_{c}=60,000$

The flow around the SD7003 airfoil at $\alpha=4^{\circ}$ and $R e_{c}=60,000$ has been studied extensively both experimentally $[37,39,40]$ and computationally $[11,12,18,23,51]$. It is generally accepted that, at low $R e_{c}$, ambient turbulence $T_{u_{\mathrm{tr}}}$ which is represented in transition criteria by $N_{c r}$ (Eq. 2) strongly influences the LSB. Hain et al. [39, Fig. 1, p. 132] evaluated, using XFoil [8, 59] calculations, the strong influence of $N_{c r}$, observing a slight delay in separation and a faster reattachement with increasing $T_{u_{\mathrm{tr}}}\left(\right.$ decreasing $\left.N_{c r}\right)$; the LSB disappears for $N_{c r} \lesssim 5.5 \stackrel{(2)}{\Longleftrightarrow} T_{u_{\mathrm{tr}}} \gtrsim 0.56 \%$.

This sensitivity on $T_{u_{\mathrm{tr}}}$ was also noted by Ol. et al. [40] who observed, between 3 different experimental facilities, substantial dispersion in the LSB location (attributed to possible minor variations of $T_{u_{\mathrm{tr}}}$ ) and flow structure (attributed to insufficient spatiotemporal resolution of the measurements). To avoid the issue of experimental discrepancies, the present RANS computations are compared with the LES results by Catalano and Tognaccini [43] and by Galbraith and Visbal [44], which are in satisfactory agreement with measurements [37, Fig. 8, p. 7]. 

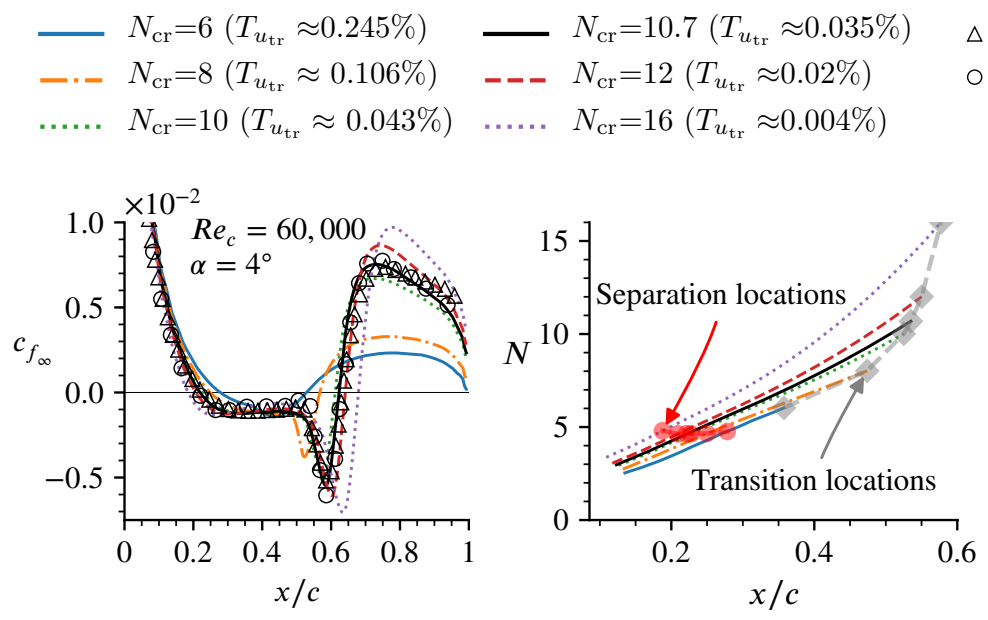
LES Catalano and Tognaccini
ILES Galbraith and Visbal

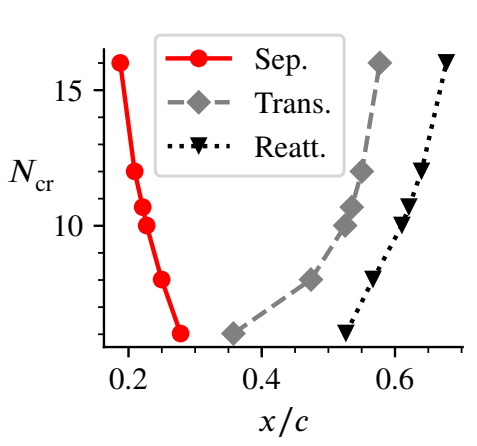

Fig. 3 Influence of external turbulence, $T_{u_{\mathrm{tr}}}$, on the suction-side $c_{f_{\infty}}$ and on separation, transition and reattachment locations; using $k-\omega[53]+$ LSTT [26] compared to LES data [43, 44].

In line with the XFoil results [39, Fig. 1, p. 132], the LSB predicted by the $k-\omega[53]+$ LSTT [26] computations at $\alpha=4^{\circ}$ and $R e_{c}=60,000$ (Fig. 3) is particularly sensitive to the value of $N_{c r}$, with a very long LSB for quiet external flow $\left(N_{c r}=16 \stackrel{(2)}{\Longleftrightarrow} T_{u_{\mathrm{tr}}} \gtrsim 0.004 \%\right)$ progressively shrinking with increasing ambient turbulence. With decreasing $N_{c r}$ (increasing $T_{u_{\mathrm{tr}}}$ ) the reattachment point moves upstream and, inversely, the laminar separation point moves downstream, leading to an overall reduction of the bubble size. The streamwise evolution of the total amplification factor $N$, on the upper side of the airfoil, predicted by Gleyzes' (§IIB) criterion (Fig. 3) highlights the influence of $N_{c r}$ on the LSB, demonstrating the impact of predicted transition onset location and of turbulence triggering on the laminar region of the flow. Obviously, the size of the LSB influences the separation location. With increasing $N_{c r}$ (decreasing $T_{u_{\mathrm{tr}}}$ ) not only the bubble but also the separated laminar region becomes larger. This implies that velocity profiles of the laminar region of the bubble yield comparatively higher $H$ values. As local stability analysis of velocity profiles show [28], these profiles are more unstable, which promotes a more rapid growth of pre-transitional perturbations, yielding comparatively higher values of $N$ as shown in Fig. 3.

The closest agreement with LES data [43, 44] is obtained with $N_{c r}=10.66 \stackrel{(2)}{\Longleftrightarrow} T_{u_{\mathrm{tr}}} \gtrsim 0.035 \%$ (Fig. 3). This value is higher than the usual range $N_{c r} \in[7,9]$ typically used for calm atmosphere conditions [59]. One possible explanation is the fact that the LES calculations [43, 44] were run 


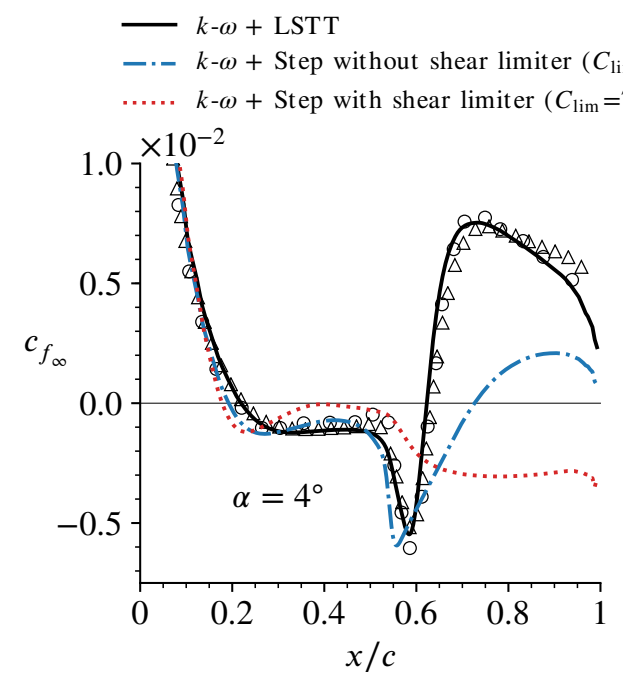

$\triangle \quad$ LES Catalano and Tognaccini

- ILES Galbraith and Visbal

$k-\omega+$ Step with shear limiter $\left(C_{\lim }=7 / 8\right)$

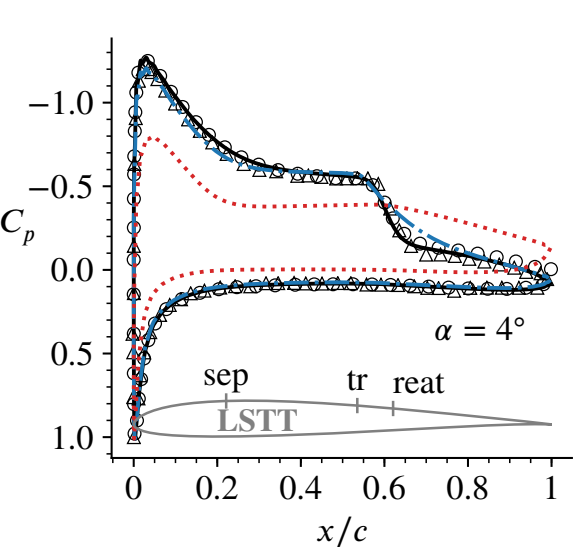

Fig. 4 Comparison of $c_{f_{\infty}}$ (on the suction side) and $C_{p}$ obtained from LES [43, 44] against $k-\omega$

[53] computations (using LSTT [26] or step-activation) for $\alpha=4^{\circ}, R e_{c}=60,000, T_{u_{\mathrm{tr}}}=0.035 \%$.

without any kind of turbulence forcing, so that the perturbations which lead to turbulence are very low, exclusively associated with numerical noise. Unless otherwise specified, the computations presented in this work used $N_{c r}=10.66$.

The particular way turbulence is triggered at transition onset has a major influence on the LSB prediction (Fig. 4). A simple off-on (§II C1) step-activation of the production terms fails to correctly reproduce the LSB. For comparison purposes the off-on computations were step-activated at $x_{t r}=0.54 c$ which is the transition onset location predicted by the $k-\omega[53]+$ LSTT [26] model. The $k-\omega[53]$ model, step-activated (§II C 1) at transition onset, with $C_{\lim }=\frac{7}{8}$, fails to correctly reproduce the LES-predicted LSB (Fig. 4), returning an open bubble that does not reattach on the profile, inducing airfoil stall. This problem is partly fixed by disabling the shear limiter in the step-activated $k-\omega[53]$ model $\left(C_{\lim }=0\right)$, predicting a bubble, but with delayed reattachment and a substantial underestimation of $c_{f_{\infty}}$ in the attached turbulent boundary-layer (Fig. 4), and failing to reproduce the very steep pressure recovery in the transitional region. The bubble-adapted LSTT model [26] gives the best agreement with LES data, both in $c_{f_{\infty}}$ and $C_{p}$ (Fig. 4), predicting the correct reattachment location and $c_{f_{\infty}}$ level downstream. As the transitional part of the bubble is better predicted by the $k-\omega[53]+$ LSTT [26] model, the boundary-layer displacement feedback on the outer flow [8] is improved, yielding also a better prediction of the location of laminar separation. 

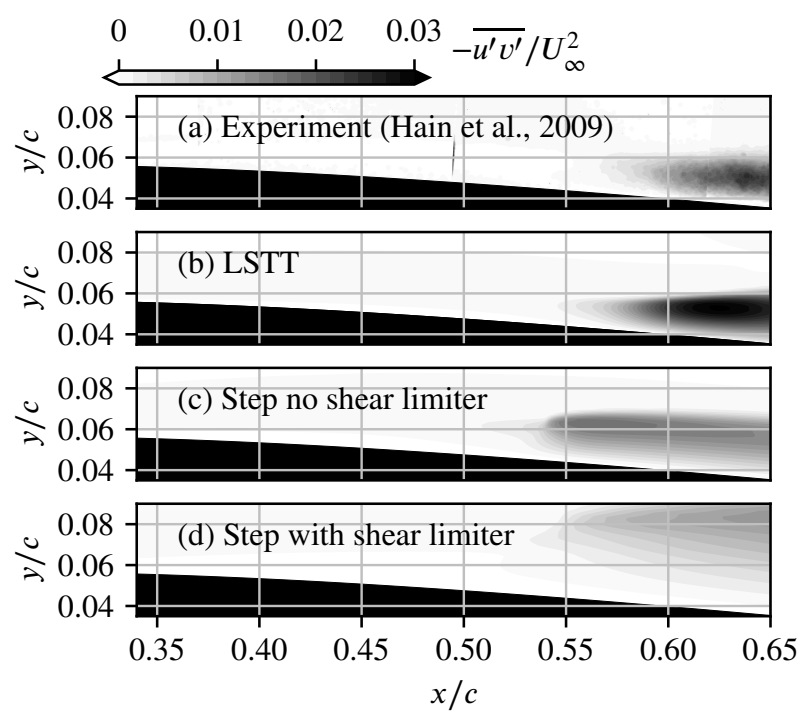

Fig. 5 Levels of Reynolds shear-stress of (a) wind-tunnel experiment [39] (Re $=66,000)$; (b-c) $k-\omega$ [53] $\left(R e_{c}=60,000\right)$ using different activations, (b) LSTT [26], (c) Step with shear limiter and (d) Step without shear limiter.

The undesired effect of the standard (fully turbulent) shear-stress limiter on the low-Reynolds number prediction of flows involving LSBs has been identified by several authors [18]. For example, the $k-\omega$ SST-LR model of Catalano and Tognaccini [18], which performs a relaxation of the SST limiter via a Reynolds-dependent weighting of Menter's second blending function $F_{2}$, predicts the correct turbulent reattachment point and $C_{p}$ distribution, but still underestimates $c_{f_{\infty}}$ downstream of the bubble [18, Fig. 15, p. 1682].

The correct prediction of $c_{f_{\infty}}$ downstream of the bubble is a difficult challenge for RANS methods. Its underestimation is the result of the underestimation of the Reynolds shear-stress $-\overline{u^{\prime} v^{\prime}}$ in the transitional shear-layer [26], leading to a convection effect that translates into a slower turbulence development and comparatively lower values of $c_{f_{\infty}}$ downstream of the bubble. This was a key issue in the development of the LSTT model [26], and is illustrated by comparing levels of measured [39] and levels of Reynolds shear-stress $-\overline{u^{\prime} v^{\prime}}$ in the LSB (Fig 5) and streamwise velocity $\bar{u}_{s}$ profiles (Fig. 6). Using step-activation with the fully turbulent $C_{\lim }=\frac{7}{8}$ severely underestimates $-\overline{u^{\prime} v^{\prime}}$ compared to the experimental data (Fig 5), and leads to unphysically excessive separation (Fig. 6). This is partly corrected by deactivating $C_{\lim }$ in the step-activated computations, but predicted levels of $-\overline{u^{\prime} v^{\prime}}$ are still underestimated (Fig 5) leading to an overestimation of bubble size (Figs. 4, 6). The 


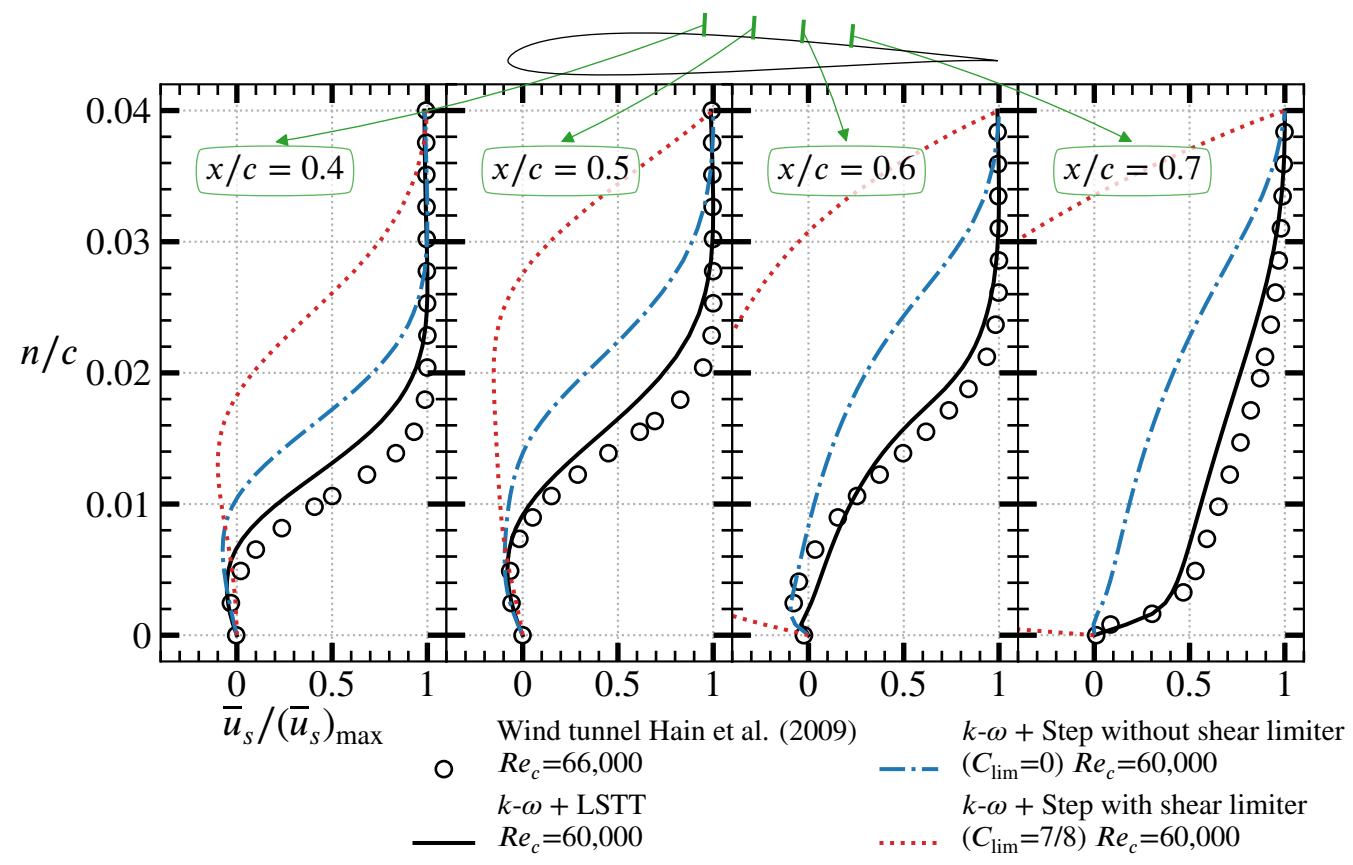

Fig. 6 Tangential velocity profiles of wind-tunnel experiment [39] $\left(R e_{c}=66,000\right)$ against $k-\omega$ [53] $\left(R e_{c}=60,000\right)$ using LSTT [26], or Step activation both with shear limiter and without.

LSTT model is necessary to correctly predict $-\overline{u^{\prime} v^{\prime}}$, which is actually slightly overestimated with comparison to the measurements (Fig 5), and as a result predict the correct bubble size (Fig. 4) and boundary-layer development (Fig. 6).

\section{B. Effect of the angle of attack at $R e_{c}=60,000$}

The $k-\omega[53]+$ LSTT [26] model is compared with LES data [43, 44] and results with the stepactivated approach (which does not include an overshoot in $\mathcal{P}_{k}$ ) for various $\alpha$ at $R e_{c}=60,000$. The LSTT model used $N_{c r}=10.66$ in the transition criteria ( $§ I I$ B) while the step-activated computations assume transition onset at the location predicted in the LSTT computations. With increasing $\alpha$ (Fig. 7), the bubble shrinks from $\sim 55 \% c$ at $\alpha=2^{\circ}$ (separation at $x \approx 0.4 c$ with reattachment at $x \approx 0.95 c$ very near the trailing-edge) to $\sim 20 \% c$ at $\alpha=11^{\circ}$ (separation very near the leading edge with reattachment at $x \approx 0.20 c)$.

Regarding the importance of the triggering approach, the conclusions are quite similar to those drawn for the baseline case $\left(\alpha=4^{\circ}, R e_{c}=60,000 ; \S I I I A\right)$. Abrupt activation of the triggering approach with fully turbulent $C_{\lim }=\frac{7}{8}$ generally fails, invariably returning stalled flow with an open 


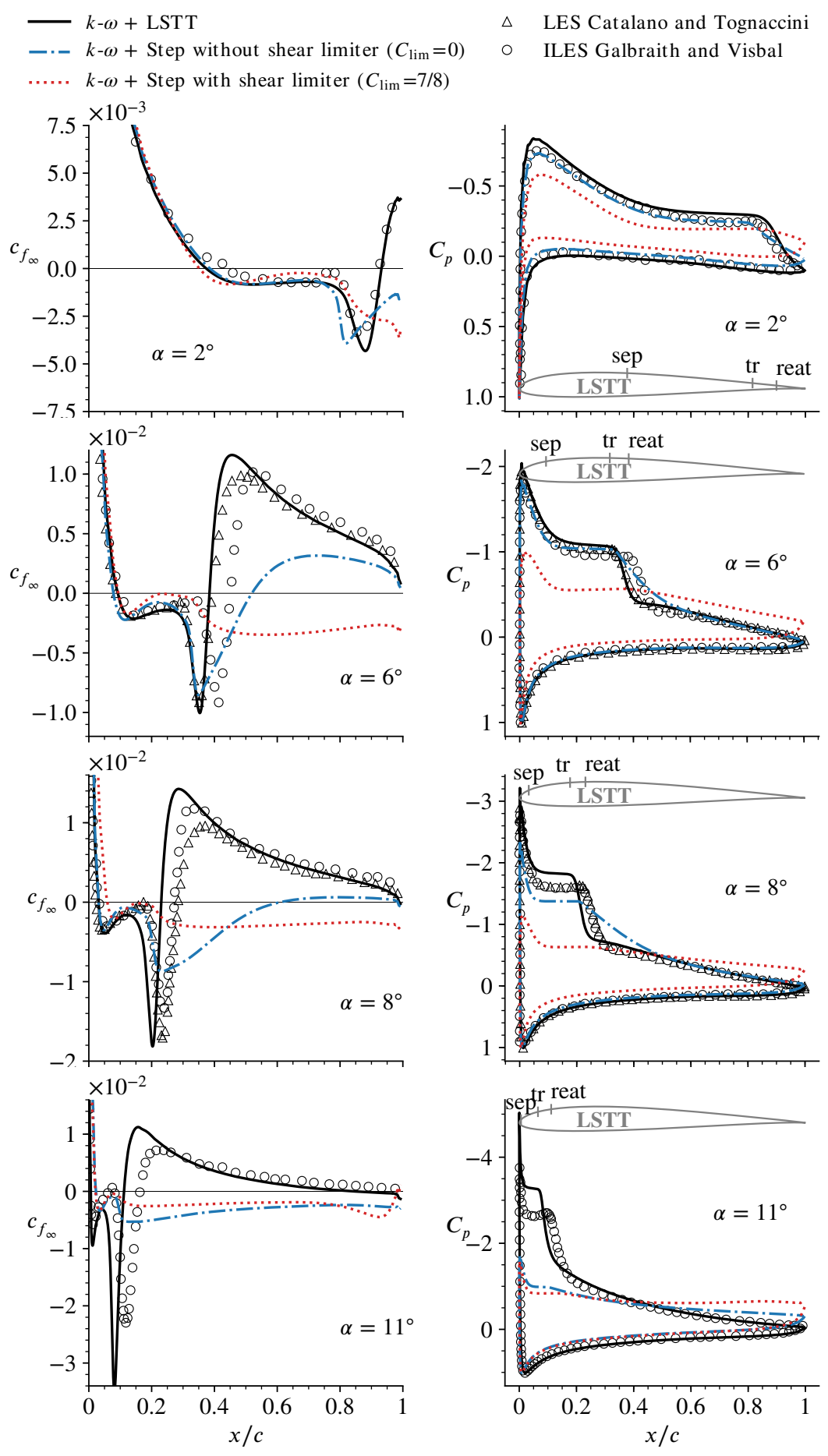

Fig. 7 Comparison of $c_{f_{\infty}}$ (on the suction side) and $C_{p}$ of LES [43, 44] against $k-\omega[53]$ using LSTT [26] and Step-activation (both with and without shear limiter) for $\left(\alpha=2^{\circ}, 6^{\circ}, 8^{\circ}, 11^{\circ}\right)$ at $R e_{c}=\mathbf{6 0 , 0 0 0}$ and $N_{\mathrm{cr}}=10.7\left(T_{u_{\mathrm{tr}}} \approx \mathbf{0 . 0 3 5 \%}\right)$. 

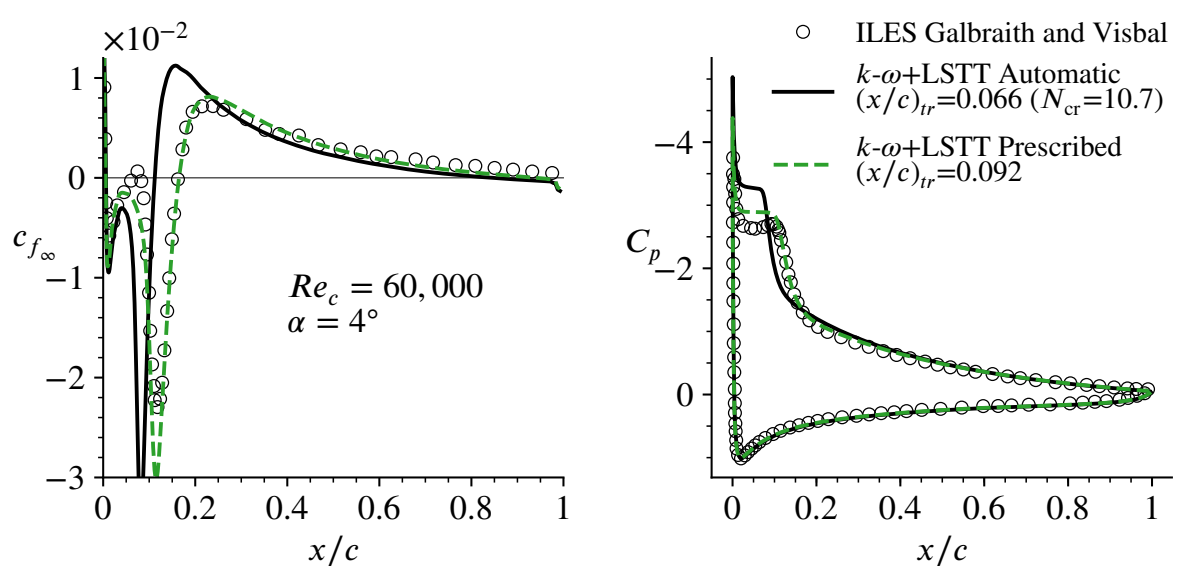

Fig. 8 Comparison of $c_{f_{\infty}}$ (on the suction side) and $C_{p}$ obtained from LES [44] against RANS computations $(k-\omega[53]+$ LSTT [26]) using automatic or prescribed transition onset location.

bubble (Fig. 7). Deactivating $C_{\lim }=0$ the shear limiter in the $k-\omega$ computations always improves the results, because, following the arguments in $§ I I I A$, the absence of the shear limiter returns a higher Reynolds shear-stress $-\overline{u^{\prime} v^{\prime}}$; nonetheless, the agreement with the LES data is still not satisfactory (Fig. 7). Although the step-activated $C_{\lim }=0 k-\omega$ method correctly predicts the initial part of the bubble, it generally underestimates the negative $c_{f_{\infty}}$ peak (increasingly so with increasing $\alpha$ ), returns an open bubble at $\alpha=2^{\circ}$, stalls at $\alpha=11^{\circ}$ or reattaches too far downstream for $\alpha \in\left\{4^{\circ}, 6^{\circ}, 8^{\circ}\right\}$ (Figs. 4,7$)$.

On the contrary, the LSTT computations are generally in satisfactory agreement (Fig. 7) with LES data [43, 44]. At $\alpha=2^{\circ}$, the agreement with the LES data of Galbraith and Visbal [44] is excellent. At $\alpha=6^{\circ}$, there is a slight scatter between the 2 sets of LES data [43, 44], the $k-\omega+$ LSTT computations being in close agreement with Catalano and Tognaccini [43]. At $\alpha=8^{\circ}$, where the 2 sets of LES data are in better agreement one with another, the $k-\omega+$ LSTT model suffers from the prediction by the criteria $(\S \mathrm{IIB})$ of too early a transition-onset location, which induces an earlier negative $c_{f_{\infty}}$ peak and earlier reattachment, also resulting in a slight discrepancy of the pressure plateau associated with the LSB (Fig. 7). The same remarks apply to the $\alpha=11^{\circ}$ case (Fig. 7). This effect was also observed [26] in the prediction of the $\alpha=10.55^{\circ}$ NACA 0012 flow at $R e_{c}=100,000$.

In order to determine the reasons for the observed discrepancies between the $k-\omega+\mathrm{LSTT}$ 
predictions and the LES data $[43,44]$ at the higher $\alpha \in\left\{8^{\circ}, 11^{\circ}\right\}$ (Fig. 7) we performed an additional calculation at $\alpha=11^{\circ}$ (Fig. 8), where the transition onset was prescribed at $x_{t r}=0.092 c$ further downstream of the criteria-predicted $\left(N_{c r}=10.66\right)$ location of transition onset at $x_{t r}=0.066 c$. This ad hoc adjustment of the transition onset location greatly improves the agreement with LES: the location of the negative $c_{f_{\infty}}$ peak and of reattachment are in excellent agreement with the LES computations, and this results in a substantially improved prediction of the pressure plateau in the $C_{p}$ distributions (Fig. 8).

These results demonstrate that the LSTT approach is quite successful in controlling the turbulence built-up in the transitional region, provided the correct $x_{\text {tr }}$ is obtained by the transition criteria. Inversely, the increased discrepancy of the $k-\omega+$ LSTT computations with LES as $\alpha$ increases is principally associated with the inaccurate (early) prediction of transition onset. This would imply that with increasing $\alpha, N_{c r}$ should also increase. Mack's correlation would then suggest that the effective $[61,62] T_{u_{\mathrm{tr}}}$ decreases. An argument in support of this observation is that as the laminar separation point moves upstream, nearer to the stagnation point, with increasing $\alpha$, the boundarylayer thickness at separation $\delta_{\text {sep }}$ is modified, and so does the receptivity of the boundary-layer to a given spectrum of external turbulence, in line with theoretical arguments [61,62] corroborated by numerical stability calculations [67]. This is naturally incorporated in LES dynamics but not in Mack's correlation (Eq. 2) which assumes a monotonic bijection $N_{c r}\left(T_{u_{\mathrm{tr}}}\right)$.

With regard to the previous results (Figs. 7, 8), it may be surmised that, as $\alpha$ increases and the LSB size shrinks, increased diffusion of momentum by the Reynolds shear-stress $-\overline{u^{\prime} v^{\prime}}$ is necessary to compensate the high inertial forces of the flow and the increased deceleration associated with the adverse local pressure gradient. It is precisely because the LSTT approach, by boosting production of $k$ and inhibiting the shear limiter until after fully turbulent conditions are reached, achieves a better prediction of the Reynolds shear-stress $-\overline{u^{\prime} v^{\prime}}$ that it performs better than the stepactivated approaches, significantly improving the predictive accuracy of the bubble's topology. The convection effect of the correct prediction of the transitional region in the LSTT approach translates into proper estimation of the $c_{f}$ values downstream of the bubble. For these reasons we will not consider step-activation in the remainder of the paper. On the other hand, it appears (Figs. 7, 8) 
that an $\alpha$-varying $N_{c r}$ input to the transition criteria ( $\left.\S \mathrm{II} \mathrm{B}\right)$ is required to handle with accuracy the prediction of transition onset.

\section{Effect of the chord-based Reynolds number}

We compare (Fig. 9) $k-\omega[53]+$ LSTT [26] computations with ILES data of Galbraith and Visbal [44] for varying $R e_{c}$ at 2 different $\alpha \in\left\{4^{\circ}, 8^{\circ}\right\}$. Expectedly, the LES data indicate that, at constant angle of attack, for increasing chord-based Reynolds number, the bubble size decreases, and the negative and positive peaks of $c_{f_{\infty}}$ become more pronounced (Fig. 9). The LSTT computations correctly capture these trends (Fig. 9).

The agreement with LES is very satisfactory for $\alpha=4^{\circ}$ (Fig. 9). Specifically, at $R e_{c}=40,000$ a larger bubble is correctly predicted compared to the $R e_{c}=60,000$ case, as transition onset has been naturally delayed downstream, and the separated laminar region has become longer. The deeper negative peak of $c_{f_{\infty}}$, as $R e_{c}$ decreases from 60,000 to 40,000 is also correctly predicted by the LSTT model (Fig. 9). Finally, at the lower $R e_{c}=10,000$, the LSTT model correctly predicts the open-bubble topology, as the flow fails to reattach before reaching the trailing-edge (Fig. 9), because in this massively laminar case transition onset occurs very close to the trailing-edge. The

$C_{p}$ distributions, at $\alpha=4^{\circ}$, are also correctly predicted by the method for all of the $3 R e_{c}$ values (Fig. 9).

At $\alpha=8^{\circ}$ the LSTT computations were also run with $N_{c r}=10.66$, although as discussed in $\S$ III B, we should have used a higher value of $N_{c r}$ to correctly predict transition onset in this case similar to the $\alpha=11^{\circ}$ case (Fig. 8). The resulting discrepancies in the prediction of transition onset by the criteria (§II B) notwithstanding, LES trends are correctly predicted by the LSTT model (Fig. 9 ), at these stronger adverse pressure gradient conditions compared to $\alpha=4^{\circ}$. For all of the $3 R e_{c}$ studied at $\alpha=8^{\circ}$ (Fig. 9) laminar separation occurs practically at the same location, very close to the leading edge, transition onset and as a consequence reattachment moving downstream with decreasing $R e_{c}$ (the pressure gradient at the end of the plateau becomes steeper with increasing $R e_{c}$ ). The LSTT model correctly predicts these trends (Fig. 9), and adjusting $N_{c r}$ for the $\alpha=8^{\circ}$ (not shown) can substantially improve the agreement with LES data, both for skin friction and 

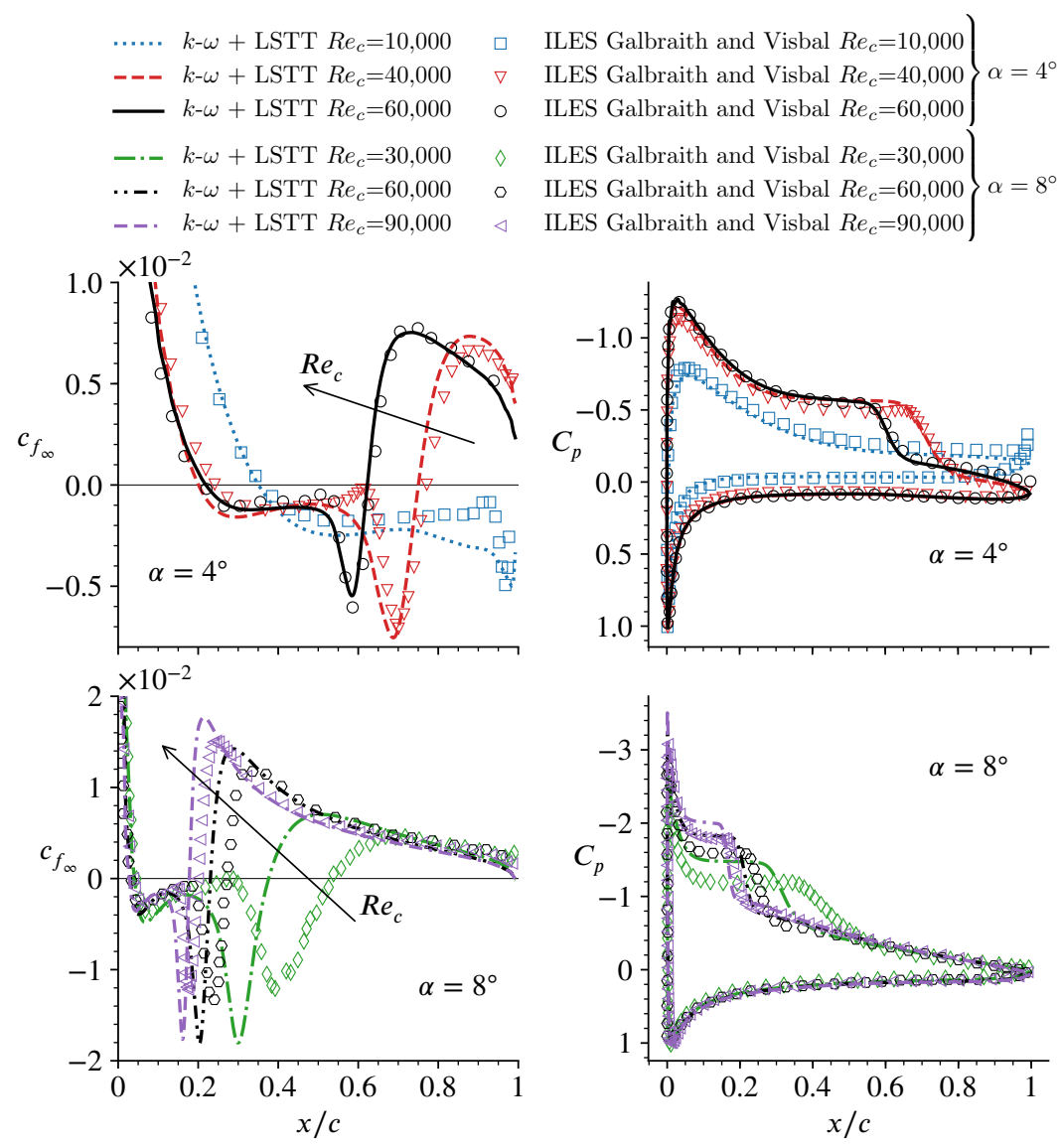

Fig. 9 Comparison of $c_{f_{\infty}}$ (on the suction side) and $C_{p}$ of LES [44], against $k-\omega$ [53] using LSTT [26] with $N_{\mathrm{cr}}=10.66$ at $\left(\alpha=4^{\circ}, 8^{\circ}\right)$ for several $R e_{c}$.

regarding the level of the pressure plateau.

\section{Effect of the Turbulence Intensity}

From the previous results and discussions it appears that the LSTT model performs generally quite well if the value of $N_{c r}$, presumably correlated to the level of external-flow turbulence at transition onset $T_{u_{t r}}$ (Eq. 2), necessary as an input to the transition criteria (§II B), is appropriately chosen. To further investigate the performance of the method under varying ambient turbulence we compare $k-\omega[53]+$ LSTT [26] computations with the LES results of Breuer [47], where the synthetic turbulence inflow generator (STIG) approach was employed in order to investigate the influence of the inflow turbulence upon the bubble topology on the SD7003 airfoil. In the LES computations, a STIG region was located one chord upstream of the airfoil, and varying the generated turbulence levels $\left(T_{u_{\text {gen }}}\right)$ with the same integral time scale $\mathcal{T} \cdot u_{\infty} / c=\mathcal{L}_{x} / c=0.118$, and 
Table 4 Synthetic turbulence level generated in the LES of Breuer [47] at $x / c=-1$, and the threshold values used in the AHD-Gleyzes criterion for the $k-\omega[53]+$ LSTT [26] RANS simulations (Fig. 10).

\begin{tabular}{lccc}
\hline \hline LES generated turbulence, $T_{u_{\text {gen }}}$ & $0 \%$ & $0.7 \%$ & $1.4 \%$ \\
\hline LSTT transition threshold, $T_{u_{\mathrm{tr}}}$ & $0.0038 \%$ & $0.070 \%$ & $0.106 \%$ \\
$N_{\mathrm{cr}}=-2.4 \ln \left(T_{u_{\mathrm{tr}}}\right)-8.43$ & 16 & 9 & 8 \\
\hline \hline
\end{tabular}

assuming the streamwise integral lengthscale $\mathcal{L}_{x}$ from Taylor's hypothesis.

It is expected [65] that the turbulence level from the STIG region $\left(T_{u_{\text {gen }}}\right)$ will decrease downstream to the airfoil's boundary-layer edge levels $\left(T_{u_{\delta}}(s)\right)$, which by Mack's correlation (Eq. 2) should provide $T_{u_{t r}}$ and $N_{c r}$. Nonetheless, the analytical evaluation of this decrease requires as input the $k-\omega$ lengthscale $\ell_{t}:=\sqrt{k} / \beta_{k} / \omega$ [65, Eqs. 14-16, p. 1103]. Notice that the exact relation between the integral lengthscale $\mathcal{L}_{x}$ and the $k-\omega$ lengthscale $\ell_{t}:=\sqrt{k} / \beta_{k} / \omega$ is not exactly known even for the simplest case of grid turbulence $[68,69]$. As a consequence, the actual $T_{u_{\delta}}(s)$ values of the LES, which should be used to determine $T_{u_{t r}}$ in the transition prediction criteria, are unknown to the authors. For this reason, with the objective to assess the predictive quality of the triggering model for varying turbulence levels, we rely on the parametric study (Fig. 3) on the effect of $N_{\mathrm{cr}}$ on the LSB for the baseline case $\left(\alpha=4^{\circ}, R e_{c}=60,000 ; \S I I I A\right)$.

The values of $N_{c r}$ used in the RANS computations and the corresponding, by Mack's correlation (Eq. 2), values of $T_{u_{t r}}$, were chosen (Tab. 4) to obtain the correct LSB size for each $T_{u_{g e n}}$ in the LES simulations [47]. The variation of turbulence level for the RANS computations was performed by direct modification of the criteria transition prediction threshold $T_{u_{\mathrm{tr}}}$, and not by modification of the boundary-layer external turbulence kinetic energy $\left(k_{\delta}\right)$, which would have required [16] appropriate modification of the freestream $k$ intensity $\left(k_{\infty}\right)$ and/or of the minimum cut-off value of $k$.

Notice first (Fig. 10) that, in the absence of synthetic turbulence inflow generation $\left(T_{u_{\text {gen }}}=0 \%\right)$, the $c_{f_{\infty}}$ and $C_{p}$ distributions obtained in the LES computations of Breuer [47] do not coincide with the previously used LES data of Catalano and Tognaccini [43] and of Galbraith and Visbal [44] (which are in close agreement one with another). This may be related to the inherent difficulty of the LES to properly account for the pre-transitional waves that lead to the transition. If no 


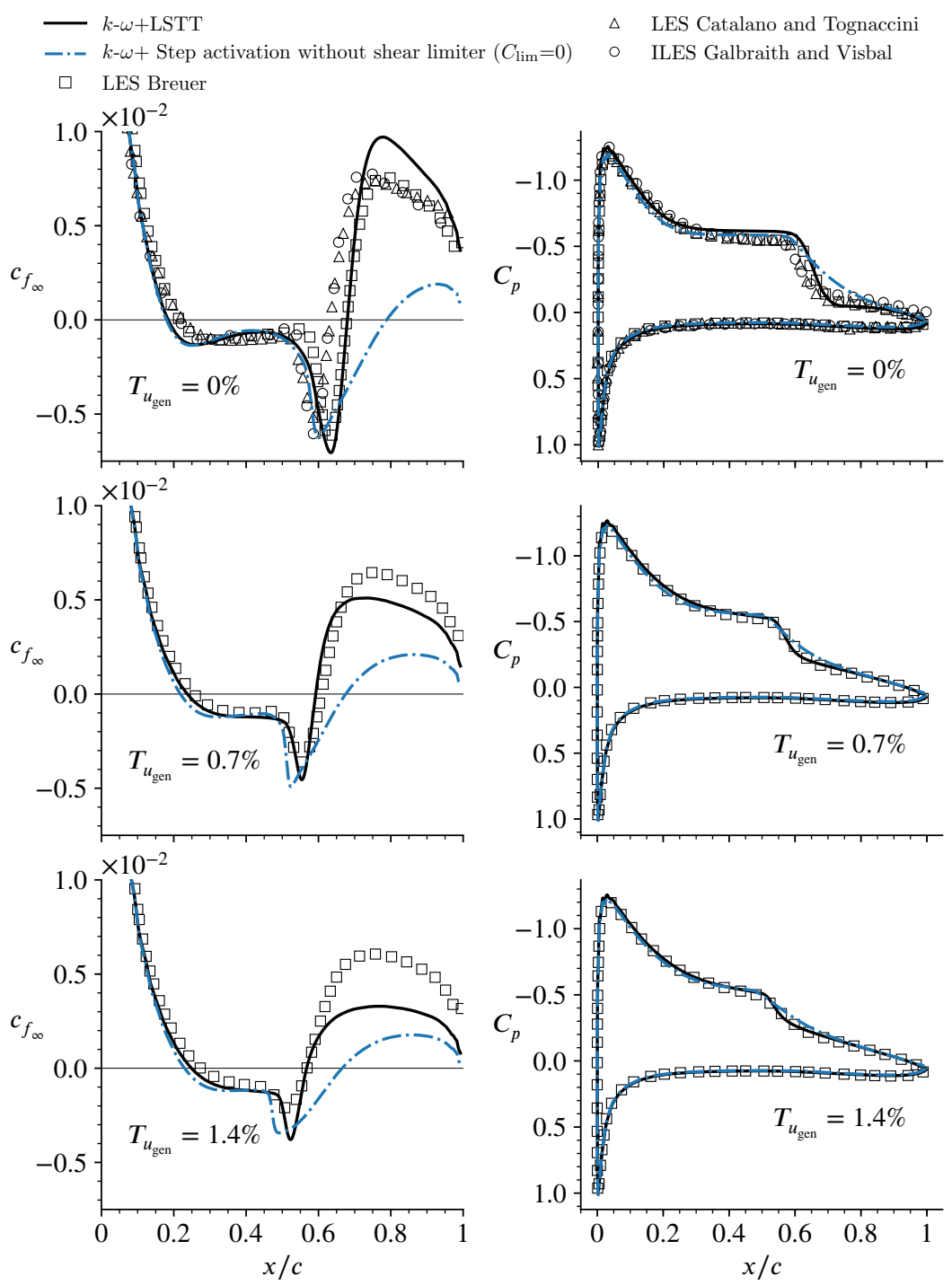

Fig. 10 Comparison of $c_{f_{\infty}}$ (on the suction side) and $C_{p}$ of LES [47], against $k-\omega$ [53] using LSTT [26] and Step activation without shear limiter at $\left(\alpha=4^{\circ}, R e_{c}=60,000\right)$ for different $T_{u}$ levels (Tab. 4).

specific forcing nor synthetic turbulence is generated, then most LES simulations rely exclusively on numerical or grid-induced errors as the only pre-transitional disturbance generator. As physically the bubble size is dependent on the inflow disturbances [70], it seems plausible that different LES run with different numerical schemes and grids without synthetic turbulence inflow generation may lead to slightly different bubble sizes.

In line with the previous results in this paper, step-activation triggering predicts a longer separation bubble and significantly underestimates $c_{f_{\infty}}$ downstream of the bubble, compared to the 


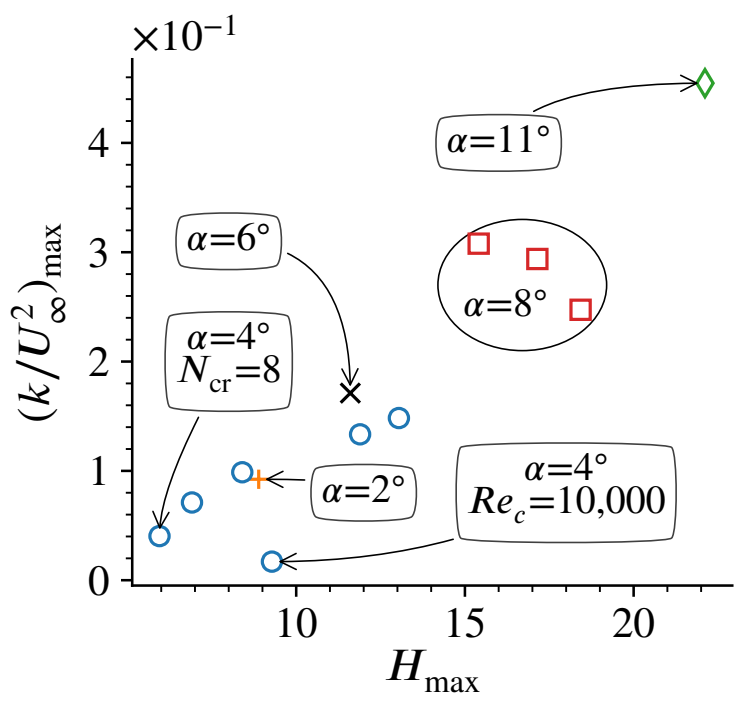

Fig. 11 Maximum values of $k / U_{\infty}^{2}$ and $H=\delta^{*} / \theta$ for all the flow configurations evaluated in the present study (varying $\alpha, R e_{c}$ and $T_{u_{\mathrm{tr}}}$ ) using the $k-\omega$ [53] +LSTT [26] model.

LES data (Fig. 10), this discrepancy increasing with increasing $T_{u_{\text {gen }}}$, contrary to the prediction of $C_{p}$ which is improved. The LSTT approach invariably improves the agreement with LES compared to the step-activation method, constantly predicting the correct size of the LSB. Nonetheless, although LSTT-predicted $C_{p}$ distributions are always in excellent agreement with LES, the level of $c_{f_{\infty}}$ downstream of the bubble is increasingly underestimated with increasing $T_{u_{\text {gen }}}$ (Fig. 10), but much less than in the step-activated computations.

The significant underestimation of skin-friction downstream of the bubble predicted by the LSTT model for the $T_{u_{\text {gen }}}=1.4 \%$ case (Fig. 10) has not been observed for any of the other flow configurations considered in the present study. This underestimation may be caused by insufficient production of Reynolds shear stress. In order to evaluate this effect, we compare the maximum values of the shape factor and the turbulence kinetic energy retrieved from all the considered cases (Fig. 11). The $H_{\max }$ value, which is located near the transition region, measures the intensity of detachment of the laminar boundary-layer, and hence is a good indicator of the transition region's mean strain rate. The $k_{\max }$ value, which is also located near the transition region, measures the intensity of turbulence, and is therefore a good indicator of the turbulence model's response to the mean strain rate near the transition region.

Expectedly the results (Fig. 11) show that an increase of $H_{\max }$ is followed by an augmentation 
of $k_{\max }$. The $\alpha=11^{\circ}$ case yields the highest values of $H_{\max }$ and $k_{\max }$, and the resulting bubble

(Fig. 8) exhibits intense skin-friction peaks. This means that a high value of $H_{\max }$, which indicates high values of $\bar{S}_{x y}$ in the detached boundary-layer, translates into a high value of $k_{\max }$, which in turn translates into pronounced skin-friction peaks near the reattachment region. On the other hand, the lowest set of $H_{\max }$ and $k_{\max }$ values is produced for the flow case $\alpha=4^{\circ}, N_{\mathrm{cr}}=8$ that is presented in Fig. $10\left(T_{u_{\mathrm{gen}}}=1.4 \%\right.$ case $)$, where the underestimation of skin-friction downstream of the bubble is identified. For this case, the low value of $H_{\max }$ indicates that the activation of the turbulence model is comparatively less responsive to the mean strain rate than the other flow cases where $H_{\max }$ is higher. This indicates that the difficulty of the $k-\omega+$ LSTT model to predict high enough skin-friction values downstream of the bubble for this flow case, is associated with very mild conditions of laminar separation.

\section{Conclusion}

The laminar separation transition triggering (LSTT) model [26] calibrated for the Wilcox 2006 $k-\omega$ turbulence model [53] has been assessed for a collection of low chord-based Reynolds number flows around the SD7003 airfoil against published LES data. The LSTT model has been successfully coupled with the AHD-Gleyzes transition prediction criteria, which makes the overall method fully predictive, requiring as additional input the threshold value $N_{c r}$ for the transition criteria.

The LSTT model predictions have been systematically compared, for varying angles of attack, chord-based Reynolds number and inflow turbulence levels, both with LES and with a classical step triggering of the turbulence model. The predictions of the LSTT model are in close agreement with the available LES data, correctly predicting the change of the bubble's position and size for the various flow conditions that were investigated. The LSTT model very substantially improves upon the classical step-activation method, which systematically underpredicts the skin-friction values downstream of the bubble.

The use of the shear limiter in the transitional region has been confirmed as the main cause of the erroneous prediction of premature stall of the airfoil when the classical step triggering is employed, even at low angles of attack for very low $R e_{c}$, because abrupt step-activation without 
overshoot of $k$-production leads to an underestimation of the Reynolds shear stress. Production boosting in the transitional region by the LSTT model better accounts for the Reynolds shear stress built-up, resulting in better agreement with the LES data.

In summary, the LSTT model [26] produces satisfactory results at relatively low inflow turbulence levels typical of external aerodynamics, and its validity has been assessed for a wide range of angles of attack and Reynolds numbers.

Areas of improvement have also been clearly identified:

1. the only case where the $k-\omega+\mathrm{LSTT}$ method underestimates the level of skin-friction downstream of turbulent reattachment was associated with very limited (in terms of boundary-layer shape factor) laminar separation, and such situations may require improved triggering strategies.

2. it is clear that the overall predictive capability of the method hinges upon the availability of accurate criteria-based determination of transition onset and the applicability of Mack's correlation. The latter appears limited because it does not take into account the ambient turbulence lengthscale compared to the boundary-layer thickness or integral lengthscales.

\section{Appendix}

Some kind of production limiter is not uncommon in 2-equation closure, both for stability purposes [71, 72] and in specific flows such as stagnation point turbulence [73]. The purpose of the limiter $\mathcal{P}_{k}=\max \left(\mathcal{P}_{k} ; 100 \varepsilon\right)$ is to avoid an unexpected over-production of turbulence above the transitional region of the bubble, as illustrated in Fig. 12. The region marked with the fuchsia dotted ellipse highlights an unexpected generation of eddy viscosity. Indeed, we have no experimental nor numerical evidence that this region of pronounced production of turbulence shall exist [39, 44]. Therefore, we consider this region as a modeling anomaly related to the triggering of the turbulence model from the transition onset. This region should not be ignored for two main reasons: (1) An unphysical region of over-production of $\mu_{t}$ could translate into a poor modeling of the Reynolds shear stress above the bubble (interestingly, in practice the strain rate tensor $S_{i j}$ is sufficiently low where the anomaly occurs so that the real impact on the Reynolds shear stress is mitigated) 

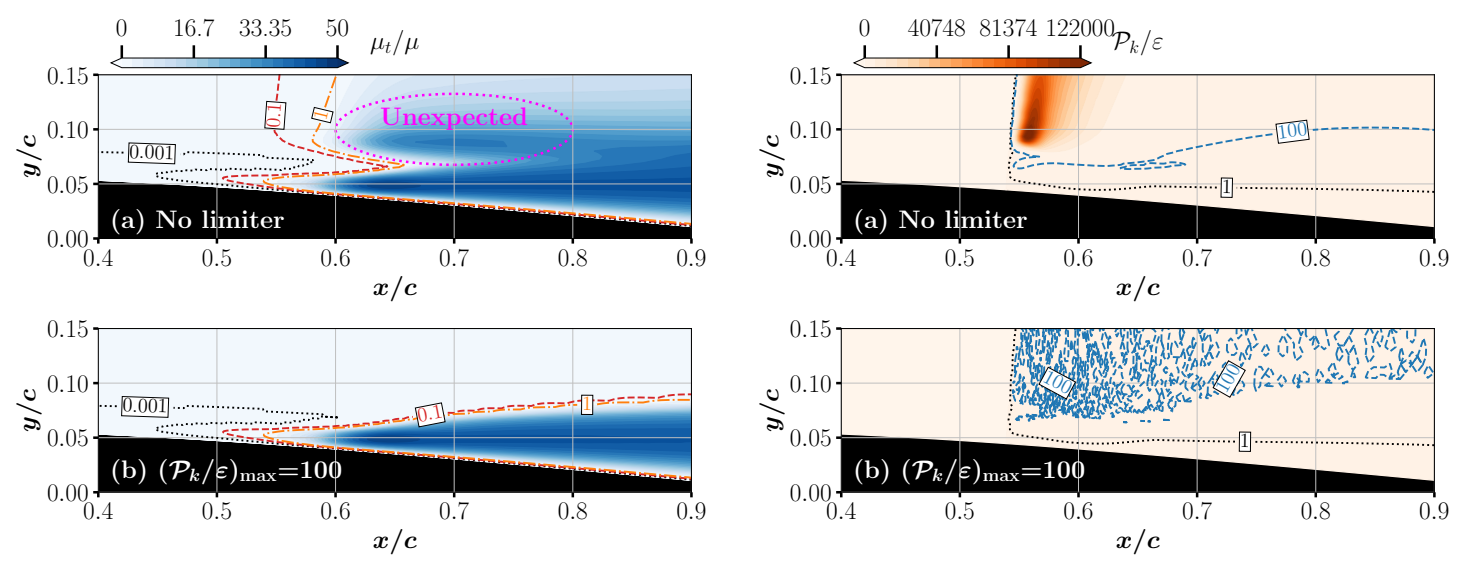

Fig. 12 Case at $\alpha=4^{\circ}$ and $R e_{c}=60,000$. Wilcox 2006 turbulence model with Bernardos et al.

[26] LSTT model. Impact of the limiter on the production-to-dissipation ratio of turbulence:

(a) Not limited, (b) $\mathcal{P}_{k} / \varepsilon$ limited to 100 .

and (2) The turbulent boundary-layer edge region should ideally yield low $\mu_{t}$ values such that the proper analytic behavior of the turbulence model is preserved $[32,74]$ and robustness of the boundary-layer is guaranteed with respect to low external disturbances [31]. Indeed, in the $\mu_{t} / \mu$ fields of Fig. 12 we have presented several contours, namely: 0.001 which is the minimum cutoff value numerically allowed; 0.1 which corresponds to the lowest value based on the freestream and turbulence quantities cut-off values $\left(\mu_{t} / \mu\right)_{\infty}=0.1$. The region delimited by the contour $\mu_{t} / \mu=0.001$ satisfactory corresponds to the laminar boundary-layer and viscous sublayer of the turbulent boundary layer. The contours $\mu_{t} / \mu=0.1$ and $\mu_{t} / \mu=1$ should be approximately situated in the turbulent/non-turbulent interface (like the boundary-layer edge) in order to guarantee proper behavior of the turbulent layer. This is clearly not the case when the limiter on the production of $k$ is disabled.

The spurious over-production of turbulence is clearly shown in the $\mathcal{P}_{k} / \varepsilon$ field of Fig. 12. Two contours are shown at $\mathcal{P}_{k} / \varepsilon=1$ and $\mathcal{P}_{k} / \varepsilon=100$. Thanks to these contours we can clearly see the saturation produced in the transition onset, situated at $x / c=0.54$, as the contours tend to merge and become normal respect to the airfoil wall. When the limiter is disabled, a high net production of turbulence is locally produced and contaminates the flowfield by classical diffusion and convection mechanisms. Limiting production-to-dissipation ratio to $\left(\mathcal{P}_{k} / \varepsilon\right)_{\max }=100$ effectively removes the 
anomaly.

\section{Acknowledgments}

The authors would like to acknowledge the great support and useful insights of our colleagues at the DAAA and DMPE Departments at ONERA. Special thanks to Professor Mr. Breuer from the Helmut-Schmidth University of Hamburg for sharing his LES results with us. We also thank Mr. Hain, Mr. Kähler and Mr. Radespiel for sharing their PIV data with us. The simulations run in this study were executed using the software elsA, which is issued of the three-party agreement between AIRBUS, SAFRAN, and ONERA. This work was completely funded by ONERA in the framework of the $\mathrm{PhD}$ thesis of the first author.

\section{References}

[1] Selig, M. S., Low Reynolds Number Airfoil Design Lecture Notes, VKI, Rhode-Saint-Genèse, Belgium, 2003.

[2] Selig, M. S., "Real-Time Flight Simulation of Highly Maneuverable Unmanned Aerial Vehicles," Journal of Aircraft, Vol. 51, No. 6, 2014, pp. 1705-1725, doi:10.2514/1.C032370.

[3] Mueller, T. J. and DeLaurier, J. D., "Aerodynamics of Small Vehicles," Annual Review of Fluid Mechanics, Vol. 35, 2003, pp. 89-111, doi:10.1146/annurev.fluid.35.101101.161102.

[4] Selig, M. S. and McGranahan, B. D., "Wind Tunnel Aerodynamic Tests of Six Airfoils for Use on Small Wind Turbines," Journal of Solar Energy Engineering, Vol. 126, 2004, pp. 986-1001, doi:10.1115/1.1793208.

[5] Selig, M. S., Guglielmo, J. J., Broeren, A. P., and Giguère, P., Summary of Low-Speed Airfoil Data, Vol. 1, SoarTech Publications, Virginia Beach, VA, 1995.

[6] Drela, M., "Low-Reynolds-number airfoil design for the M.I.T. Daedalus prototype- A case study," AIAA Journal of Aircraft, Vol. 25, No. 8, 1988, pp. 724-732, doi: $10.2514 / 3.45650$.

[7] Eppler, R., Airfoil Design and Data, Springer-Verlag, New York, 1990, doi:10.1007/978-3-662-02646-5. 
[8] Drela, M. and Giles, M. B., "Viscous-Inviscid Analysis of Transonic and Low Reynolds Number Airfoils," AIAA Journal, Vol. 25, No. 10, 1987, pp. 1347-1355, doi:10.2514/3.9789.

[9] Cliquet, J., Houdeville, R., and Arnal, D., "Application of Laminar-Turbulent Transition Criteria in Navier-Stokes Computations," AIAA Journal, Vol. 46, No. 5, 2008, pp. 1182-1190, doi:10.2514/1.30215.

[10] Wilcox, D. C., "Simulation of Transition with a Two-Equation Turbulence Model," AIAA Journal, Vol. 32, No. 2, 1994, pp. 247-255, doi: $10.2514 / 3.59994$.

[11] Windte, J., Scholz, U., and Radespiel, R., "Validation of the RANS-simulation of laminar separation bubbles on airfoils," Aerospace Science and Technology, Vol. 10, No. 6, 2006, pp. 484-494, doi:10.1016/j.ast.2006.03.008.

[12] Catalano, P. and Tognaccini, R., "RANS analysis of the low-Reynolds number flow around the SD7003 airfoil," Aerospace Science and Technology, Vol. 15, No. 8, 2011, pp. 615 - 626, doi:10.1016/j.ast.2010.12.006.

[13] Rumsey, C. L., "Apparent transition behavior of widely-used turbulence models," International Journal of Heat and Fluid Flow, Vol. 28, No. 6, 2007, pp. 1460-1471, doi:10.1016/j.ijheatfluidflow.2007.04.003.

[14] Gerolymos, G. A. and Vallet, I., "Bypass Transition and Tripping in Reynolds-stress Model Computations," in "21st AIAA Computational Fluid Dynamics Conference," AIAA, San Diego, CA, 2013, doi:10.2514/6.2013-2425.

[15] Rumsey, C. L., Thacker, W. D., Gatski, T. B., and Grosch, C. E., "Analysis of Transition-Sensitized Turbulent Transport Equations," in "43rd AIAA Aerospace Sciences Meeting and Exhibit," AIAA, Reno, Nevada, USA, 2005-0523, 2005, pp. 1-16, doi:10.2514/6.2005-523.

[16] Rumsey, C. L. and Spalart, P. R., "Turbulence Model Behavior in Low Reynolds Number Regions of Aerodynamic Flowfields," AIAA Journal, Vol. 47, No. 4, 2009, pp. 982-993, doi: $10.2514 / 1.39947$.

[17] Dick, E. and Kubacki, S., "Transition models for turbomachinery boundary layer flows : a review," International Journal Of Turbomachinery Propulsion And Power, Vol. 2, No. 2, 2017, p. 44, doi:10.3390/ijtpp2020004.

[18] Catalano, P. and Tognaccini, R., "Turbulence Modeling for Low-Reynolds-Number Flows," AIAA Jour- 
nal, Vol. 48, No. 8, 2010, pp. 1673-1685,

doi:10.2514/1.J050067.

[19] Langtry, R. and Menter, F., "Transition Modeling for General CFD Applications in Aeronautics," in "43rd AIAA Aerospace Sciences Meeting and Exhibit," AIAA, American Insitute of Aeronautics and Astronautics, Reno, Nevada, USA, 2005, doi:10.2514/6.2005-522.

[20] Langtry, R. B., Menter, F. R., Likki, S. R., Suzen, Y. B., Huang, P. G., and Völker, S., "A CorrelationBased Transition Model Using Local Variables - Part II: Test Cases and Industrial Applications," Journal of Turbomachinery, Vol. 128, 2006, pp. 423-434, doi:10.1115/1.2184353.

[21] Menter, F. R., Smirnov, P. E., Liu, T., and Avancha, R., "A One-Equation Local Correlation-Based Transition Model," Flow, Turbulence and Combustion, Vol. 95, No. 4, 2015, pp. 583-619, doi:10.1007/s10494-015-9622-4.

[22] Walters and Cokljat, "A Three-Equation Eddy-Viscosity Model for Reynolds-Averaged Navier-Stokes Simulations of Transitional Flow," Journal of Fluids Engineering, Vol. 130, No. 12, doi:10.1115/1.2979230.

[23] Lian, Y. and Shyy, W., "Laminar-Turbulent Transition of a Low Reynolds Number Rigid or Flexible Airfoil," AIAA Journal, Vol. 45, No. 7, 2007, pp. 1501-1513, doi:10.2514/1.25812.

[24] Serna, J. and Lázaro, B. J., "The final stages of transition and the reattachment region in transitional separation bubbles," Experiments in Fluids, Vol. 55, No. 4, 2014, p. 1695, doi:10.1007/s00348-014-1695-7.

[25] Arnal, D., "Laminar-turbulent transition problems in supersonic and hypersonic flow," Tech. Rep. 761, AGARD, 1988.

[26] Bernardos, L., Richez, F., Gleize, V., and Gerolymos, G. A., "Algebraic Nonlocal Transition Modeling of Laminar Separation Bubbles using k- $\omega$ Turbulence Models," AIAA Journal, Vol. 57, No. 2, 2019, pp. $553-565$, doi:10.2514/1.J057734.

[27] Arnal, D., Habiballah, M., and Coustols, E., "Laminar Instability Theory And Transition Criteria In Two and Three-Dimensional Flow," Recherche Aérospatiale (ISSN 0379-380X), Vol. 2, 1984, pp. $125-143$.

[28] Gleyzes, C., Cousteix, J., and Bonnet, J. L., "A Calculation Method of Leading-Edge Separation Bub- 
bles," in Cebeci, T., ed., "Numerical and Physical Aspects of Aerodynamic Flows II," Springer Berlin Heidelberg, Berlin, Heidelberg, 1984, pp. 173-192,

doi:10.1007/978-3-662-09014-5_10.

[29] Wilcox, D. C., "Reassessment of the scale-determining equation for advanced turbulence models," AIAA Journal, Vol. 26, No. 11, 1988, pp. 1299-1310, doi:10.2514/3.10041.

[30] Menter, F. R., "Two-equation eddy-viscosity turbulence models for engineering applications," AIAA Journal, Vol. 32, No. 8, 1994, pp. 1598-1605, doi: $10.2514 / 3.12149$.

[31] Kok, J., "Resolving the Dependence on Freestream Values for the k- Turbulence Model," AIAA Journal, Vol. 38 , No. 7, 2000, pp. 1292-1295, doi:10.2514/2.1101.

[32] Wilcox, D. C., Turbulence Modeling for CFD, DCW Industries, La Cañada, California, 3rd ed., 2006.

[33] Laurent, C., Mary, I., Gleize, V., Lerat, A., and Arnal, D., "DNS database of a transitional separation bubble on a flat plate and application to RANS modeling validation," Computers 6 Fluids, Vol. 61, 2012 , pp. $21-30$, doi:10.1016/j.compfluid.2011.07.011. Onera Scientific Day.

[34] Selig, Donovan, and Fraser, Airfoils at Low-Speeds, SoarTech Aero Publications, Virginia Beach, VA, 1989.

[35] Burgmann, S. and Schröder, W., "Investigation of the vortex induced unsteadiness of a separation bubble via time-resolved and scanning PIV measurements," Experiments in Fluids, Vol. 45, No. 4, 2008, p. 675 , doi:10.1007/s00348-008-0548-7.

[36] Burgmann, S., Dannemann, J., and Schröder, W., "Time-resolved and volumetric PIV measurements of a transitional separation bubble on an SD7003 airfoil," Experiments in Fluids, Vol. 44, No. 4, 2008, pp. 609-622, doi:10.1007/s00348-007-0421-0.

[37] Olson, D. A., Katz, A. W., Naguib, A. M., Koochesfahani, M. M., Rizzetta, D. P., and Visbal, M. R., "On the challenges in experimental characterization of flow separation over airfoils at low Reynolds number," Experiments in Fluids, Vol. 54, No. 2, 2013, p. 1470, doi:10.1007/s00348-013-1470-1.

[38] Zilli, J., Sutton, D. M., and Lavoie, P., "Effect of Freestream Turbulence on Laminar Separation Bubbles 
and Flow Transition on an SD7003 Airfoil at Low Reynolds Numbers," in "55th AIAA Aerospace Sciences Meeting," Grapevine, Tewa, 2017,

doi:10.2514/6.2017-0302.

[39] Hain, R., Kähler, C. J., and Radespiel, R., "Dynamics of laminar separation bubbles at low-Reynoldsnumber aerofoils," Journal of Fluid Mechanics, Vol. 630, 2009, pp. 129-153, doi:10.1017/S0022112009006661.

[40] Ol, M. V., McAuliffe, B. R., Hanff, E. S., Scholz, U., and Kähler, C., "Comparison of Laminar Separation Bubble Measurements on a Low Reynolds Number Airfoil in Three Facilities," in "35th AIAA Fluid Dynamics Conference and Exhibit," Toronto, Ontario, Canada, 2005, doi:10.2514/6.2005-5149.

[41] Herbst, S., Kähler, C., and Hain, R., "Influence of large-scale free-stream turbulence on an SD7003 airfoil at low Reynolds numbers," in "AIAA AVIATION Forum," Atlanta, USA, Applied Aerodynamics Conference, 2018, doi:10.2514/6.2018-3490.

[42] Visbal, M. R., "High-Fidelity Simulation of Transitional Flows past a Plunging Airfoil," AIAA Journal, Vol. 47, No. 11, 2009, pp. 2685-2697, doi:10.2514/1.43038.

[43] Catalano, P. and Tognaccini, R., "Influence of Free-stream Turbulence on Simulations of Laminar Separation Bubbles," in "47th AIAA Aerospace Sciences Meeting Including The New Horizons Forum and Aerospace Exposition," AIAA, Orlando, Florida, USA, 2009, doi:10.2514/6.2009-1471.

[44] Galbraith, M. C. and Visbal, M. R., "Implicit Large Eddy Simulation of Low-Reynolds-Number Transitional Flow Past the SD7003 Airfoil," in "40th Fluid Dynamics Conference and Exhibit," AIAA, Chicago, Illinois, USA, 2010, doi:10.2514/6.2010-4737.

[45] Uranga, A., Persson, P.-O., Drela, M., and Peraire, J., "Implicit Large Eddy Simulation of transition to turbulence at low Reynolds numbers using a Discontinuous Galerkin method," International Journal for Numerical Methods in Engineering, Vol. 87, No. 1âĂ̌̌5, , pp. 232-261, doi:10.1002/nme.3036.

[46] Schmidt, S. and Breuer, M., "Source term based synthetic turbulence inflow generator for eddy-resolving predictions of an airfoil flow including a laminar separation bubble," Computers Es Fluids, Vol. 146, 2017, pp. $1-22$, 
doi:10.1016/j.compfluid.2016.12.023.

[47] Breuer, M., "Effect of Inflow Turbulence on an Airfoil Flow with Laminar Separation Bubble: An LES Study," Flow, Turbulence and Combustion, doi:10.1007/s10494-017-9890-2.

[48] Nguyen, L., Golubev, V. V., and Visbal, M. R., "Numerical Study of Transitional SD7003 Airfoil Interacting with Canonical Upstream Flow Disturbances," AIAA Journal, Vol. 56, No. 1, 2018, pp. $158-181$ doi:10.2514/1.J055900.

[49] McGranahan, B. D. and Selig, M. S., "Surface Oil Flow Measurements on Several Airfoils at Low Reynolds Numbers," in "21st AIAA Applied Aerodynamics Conference," AIAA, AIAA, Orlando, Florida, USA, 2003-4067, 2003, pp. 1-18, doi:10.2514/6.2003-4067.

[50] Sutton, D. M., Experimental Characterization of the Effects of Freestream Turbulence Intensity on the SD7003 Airfoil at Low Reynolds Numbers, Master's thesis, University of Toronto, Toronto, Canada, 2015.

[51] Counsil, J. N. N. and Boulama, K. G., "Low-Reynolds-Number Aerodynamic Performances of the NACA 0012 and Selig-Donovan 7003 Airfoils," Journal of Aircraft, Vol. 50, No. 1, 2013, pp. 204-216, doi:10.2514/1.C031856.

[52] Choudhry, A., Arjomandi, M., and Kelso, R., "A study of long separation bubble on thick airfoils and its consequent effects," International Journal of Heat and Fluid Flow, Vol. 52, 2015, pp. 84 - 96, doi:10.1016/j.ijheatfluidflow.2014.12.001.

[53] Wilcox, D. C., "Formulation of the $k-\omega$ Turbulence Model Revisited," AIAA Journal, Vol. 46, No. 11, 2008, pp. 2823-2838, doi: $10.2514 / 1.36541$.

[54] Bernardos, L., Richez, F., Gleize, V., and Gerolymos, G. A., "On the $k-\omega$ models behavior in the boundary-layer downstream of a short transitional separation bubble," in "AIAA Aerospace Sciences Meeting," AIAA, Kissimmee, Florida, USA, 2018, doi:10.2514/6.2018-0376.

[55] Menter, F. R., "Performance of popular turbulence model for attached and separated adverse pressure gradient flows," AIAA Journal, Vol. 30, No. 8, 1992, pp. 2066-2072, doi:10.2514/3.11180.

[56] Zheng, X. and Liu, F., "Staggered upwind method for solving Navier-Stokes and k-omega turbulence 
model equations," AIAA Journal, Vol. 33, No. 6, 1995, pp. 991-998, doi:0.2514/3.12808.

[57] Perraud, J., Deniau, H., and Casalis, G., "Overview Of Transition Prediction Tools In The elsA Software," in "6th European Conference on Computational Fluid Dynamics," IACM and ECCOMAS, HAL, Barcelona, Spain, hal-01077974, 2014.

[58] Mack, L. M., "Transition prediction and linear stability theory," Tech. Rep. CP 224, 1.1-1.22, AGARD, 1977.

[59] Drela, M., XFoil: An Analysis and Design System for Low Reynolds Number Airfoils, Springer-Verlag, 1989 , doi:10.1007/978-3-642-84010-4_1.

[60] Drela, M., "MISES Implementation of Modified Abu-Ghannam/Shaw Transition Criterion," online, 1998.

[61] Dullenkopf, K. and Mayle, R., "An account of free-stream-turbulence lengthscale on laminar heat transfer," ASME Journal of Turbomachinery, Vol. 117, 1995, pp. 401-406, doi:10.1115/94-GT-174.

[62] Mayle, R. E., Dullenkopf, K., and Schulz, A., "The Turbulence That Matters," ASME Journal of Turbomachinery, Vol. 120, No. 3, 1998, pp. 402-409, doi:10.1115/97-GT-274.

[63] Cambier, L., Heib, S., and Plot, S., "The Onera elsA CFD software: input from research and feedback from industry," Mechanics $\&$ Industry, Vol. 14, No. 3, 2013, pp. 159-174, doi:10.1051/meca $/ 2013056$.

[64] Spalart, P. and Rumsey, C. L., "Effective Inflow Conditions for Turbulence Models in Aerodynamic Calculations," AIAA Journal, Vol. 45, No. 10, 2007, pp. 2544-2553, doi:10.2514/1.29373.

[65] Gerolymos, G. A., Sauret, E., and Vallet, I., "Influence of Inflow Turbulence in Shock-Wave/TurbulentBoundary-Layer Interaction Computations," AIAA Journal, Vol. 42, No. 6, 2004, pp. 1101-1106, doi:10.2514/1.2257.

[66] Benoit, C., Péron, S., and Landier, S., "Cassiopee: A CFD pre- and post-processing tool," Aerospace Science and Technology, Vol. 45, No. Supplement C, 2015, pp. $272-283$, doi:10.1016/j.ast.2015.05.023.

[67] Vermeersch, O. and Arnal, D., "Klebanoff-Mode Modeling and Bypass-Transition Prediction," AIAA Journal, Vol. 48, No. 11, 2010, pp. 2491-2500, 
doi:10.2514/1.J050002.

[68] Valente, P. C. and Vassilicos, J. C., "The decay of turbulence generated by a class of multiscale grids," Journal of Fluid Mechanics, Vol. 687, 2011, pp. 300-340, doi:10.1017/jfm.2011.353.

[69] Valente, P. C. and Vassilicos, J. C., "The energy cascade in grid-generated non-equilibrium decaying turbulence," Physics of Fluids, Vol. 27, No. 045103, 2015, pp. 1-25, doi:10.1063/1.4916628.

[70] Yarusevych, S. and Kotsonis, M., "Steady and transient response of a laminar separation bubble to controlled disturbances," Journal of Fluid Mechanics, Vol. 813, 2017, pp. 955-990, doi:10.1017/jfm.2016.848.

[71] Gerolymos, G. A., "Implicit multiple-grid solution of the compressible Navier-Stokes equations using k-epsilon turbulence closure," AIAA Journal, Vol. 28, No. 10, 1990, pp. 1707-1717, doi: $10.2514 / 3.10464$.

[72] Gerolymos, G. A. and Vallet, I., "Implicit computation of three-dimensional compressible Navier-Stokes equations using k-epsilon closure," AIAA Journal, Vol. 34, No. 7, 1996, pp. 1321-1330, doi:10.2514/3.13236.

[73] Kato, M. and Launder, B. E., "The Modeling of Turbulent Flow Around Stationary and Vibrating Square Cylinders," in "9th Symposium on Turbulent Shear Flows," Kyoto, Japan, 1993, pp. 10-4-1 10-4-6.

[74] Ferrey, P. and Aupoix, B., "Behaviour of turbulence models near a turbulent/non-turbulent interface revisited," International Journal of Heat and Fluid Flow, Vol. 27, No. 5, 2006, pp. 831 - 837, doi:10.1016/j.ijheatfluidflow.2006.03.022. Special issue of the 6th International Symposium on Engineering Turbulence Modelling and Measurements ETMM6. 